\title{
FILTERS AND THE WEAK ALMOST PERIODIC COMPACTIFICATION OF A DISCRETE SEMIGROUP
}

\author{
BY
}

JOHN F. BERGLUND AND NEIL HINDMAN ${ }^{1}$

\begin{abstract}
The weak almost periodic compactification of a semigroup is a compact semitopological semigroup with certain universal properties relative to the original semigroup. It is not, in general, a topological compactification. In this paper an internal construction of the weak almost periodic compactification of a discrete semigroup is constructed as a space of filters, and it is shown that for discrete semigroups, the compactification is usually topological. Other results obtained on the way to the main one include descriptions of weak almost periodic functions on closed subsemigroups of topological groups, conditions for functions on the additive natural numbers or on the integers to be weak almost periodic, and an example to show that the weak almost periodic compactification of the natural numbers is not the closure of the natural numbers in the weak almost periodic compactification of the integers.
\end{abstract}

1. Introduction. A semitopological semigroup is a triple $(S,+, \mathcal{T})$ such that $(S,+)$ is a semigroup, $(S, \mathcal{T})$ is a Hausdorff topological space, and + is separately continuous. While topological semigroups (those for which + is jointly continuous) have received most of the attention over the years, it is semitopological semigroups which arise naturally in such subjects as abstract harmonic analysis and functional analysis. For example, semitopological semigroups occur as the structure space of a measure algebra, as the weak operator closure of certain semigroups of operators, as the enveloping semigroup of certain flows, and as the weak almost periodic compactification of topological groups.

We write our semigroups additively, by the way, since we shall be concerned mostly with the semigroup $(\mathbf{N},+)$ of natural numbers and the group $(\mathbf{Z},+)$ of integers.

Define a function $f$ in $C(S)$, the set of bounded continuous complex-valued functions on $S$, to be weak almost periodic on $S$ provided

$$
\lim _{n \rightarrow \infty} \lim _{k \rightarrow \infty} f\left(s_{n}+t_{k}\right)=\lim _{k \rightarrow \infty} \lim _{n \rightarrow \infty} f\left(s_{n}+t_{k}\right)
$$

whenever $\left\langle s_{n}\right\rangle_{n=1}^{\infty}$ and $\left\langle t_{k}\right\rangle_{k=1}^{\infty}$ are sequences in $S$ and all limits involved exist. (For other characterizations see Theorem 2.3.) The set

$$
W(S)=\{f \in C(S) \mid f \text { is weak almost periodic }\}
$$

is a sub- $C^{*}$-algebra of $C(S)$.

Received by the editors May 17, 1982 and, in revised form, September 16, 1982 and May 2, 1983.

1980 Mathematics Subject Classification. Primary 22A15; Secondary 43A60.

'This author gratefully acknowledges support from the National Science Foundation under grants MCS 78-02330 and MCS 81-00733. 
In $\$ 2$ we present some background about $W(S)$ and certain maximal compact extensions of $S$. One of these, denoted $\omega S$ and called the weak almost periodic compactification of $S$, has the property that exactly the members of $W(S)$ extend continuously to $\omega S$. In general, the weak almost periodic compactification is not a compactification, as the term is used in general topology; not only may the embedding function fail to be a homeomorphism, but it may not even be one-to-one. Also in $\S 2$, we give sufficient conditions for some locally compact semigroups $S$ to have $\omega S$ be a topological compactification; these conditions imply, for instance, that any closed subsemigroup $S$ of a locally compact group $G$ has $\omega S$ as a topological compactification.

The weak almost periodic compactification of a semitopological semigroup carries an algebraic structure with regard to which it is itself a semitopological semigroup. If $(S,+, \mathcal{T})$ is a triple with $(S,+)$ a semigroup, $(S, \mathcal{T})$ is a topological space, and + is "left-continuous" (meaning that for all $s \in S$, the function $t \rightarrow s+t: S \rightarrow S$ is continuous), then $(S,+, \mathcal{T})$ is a left topological semigroup. Given a semitopological semigroup $S$, there is a compact left topological semigroup associated with it much like $\omega S$ is associated with $S$. In many cases, including all discrete semigroups $S$, the space of this left topological semigroup is the Stone-Čech compactification $\beta S$ of $S$. $\$ 2$ contains more information about these compactifications.

In $\S 3$ we derive some results about $\omega S$ under various hypotheses about the semitopological semigroup $S$. The main results concern semigroups $S$ which are closed subsemigroups of locally compact topological groups. We show, for instance, that if the $C^{*}$-algebra $W(S)$ for such $S$ decomposes into a direct sum

$$
W(S)=W(S)_{0} \oplus W(S)_{p},
$$

where $W(S)_{0}$ is the closed ideal of $W(S)$ consisting of those functions with 0 in their orbits, and $W(S)_{p}$ is the subalgebra of strong almost periodic functions, then the functions in $W(S)_{0}$ can be described rather completely. (A function $f \in C(S)$ is strong almost periodic if it is the uniform limit of a sequence of coefficients of finite-dimensional unitary representations of $S$.)

In certain cases, in particular if $S$ is $(\mathbf{N},+)$ or $(\mathbf{Z},+), \omega S$ is a topological quotient of $\beta S$; a function $f \in C(S)$ is in $W(S)$ if and only if

$$
f^{\beta}(p+q)=f^{\beta}(q+p), \quad \forall p, q \in \beta S,
$$

where $f^{\beta}$ is the unique extension of $f$ to $\beta S$. Beyond $\S 3$ we restrict our attention to discrete semigroups $S$, so that $\omega S$ is a quotient of $\beta S$.

In $\S 4$ we investigate several conditions which guarantee that a function $f$ in $C(\mathbf{N})$ or $C(\mathbf{Z})$ is weak almost periodic. (We also give some criteria by which one can tell that a function is not weak almost periodic.) We also show in $\$ 4$ that not all functions in $W(\mathbf{N})$ extend as weak almost periodic functions to $\mathbf{Z}$, which implies that the weak almost periodic compactification $\omega \mathbf{N}$ of $\mathbf{N}$ is not the closure of $\mathbf{N}$ in $\omega \mathbf{Z}$. Thus, the various results in this paper about closed subsemigroups $S$ of locally compact groups $G$ are not simply inherited from $\omega G$ or $W(G)$.

We view $\beta S$, for discrete $S$, as the set of all ultrafilters on $S$. As such a (Hausdorff) quotient of $\beta S$ is a set of closed subsets of $\beta S$. The closed subsets of $\beta S$ correspond to filters on $S$. 
In $\$ 5$ we investigate compactifications of $S$ as spaces of filters, obtaining in Corollary 5.12 a description, internal to $R$, of when a set $\mathscr{R}$ of filters on $S$ yields a Hausdorff compactification of $(S,+)$. (That result does not refer to the algebraic structure of $S$.) We also obtain a characterization, internal to $R$, of when a set $R$ of filters yields a left topological semigroup compactification of $(S,+)$ (in a sense made precise in Theorem 5.18).

In $\$ 6$ we characterize $\omega S$, for discrete $S$, as a space of filters. Heretofore, all the constructions of weak almost periodic compactifications have been external to the semigroup (e.g., the closure of a set of operators among other operators or of a set of means among other means). We believe this construction, from the inside out, provides a better understanding of the structure of weak almost periodic compactifications. In particular, we apply this construction to obtain the cardinality of $\omega S$ for a large class of semigroups $S$.

2. Preliminaries. We describe here some notation and background information. Most of the results of this section are well known.

If $\phi: A \rightarrow B$ and $C \subseteq A$, we let

$$
\phi[C]=\{\phi(x) \mid x \in A\} .
$$

The set $\mathbf{N}$ is the set of positive integers $(0 \notin \mathbf{N})$, and $\mathbf{Z}$ is the set of all integers.

Given a discrete space $X$, the set

$$
\beta X=\{p \mid p \text { is an ultrafilter on } X\} .
$$

For $x \in X$, define $\hat{x}=\{A \subseteq X \mid x \in A\}$. The map $x \rightarrow \hat{x}$ embeds $X$ in $\beta X$, and we frequently identify $x$ with $\hat{x}$ (and thus pretend that $X \subseteq \beta X$ ). For $A \subseteq X$, let

$$
\bar{A}=\{p \in X \mid A \in p\} .
$$

The set $\{\bar{A} \mid A \subseteq X\}$ forms a basis for a Hausdorff topology on $\beta X$ (and a basis for the closed sets as well). With this topology $\beta X$ is the Stone-Čech compactification of $X$. That is, $\beta X$ is compact, and if $\phi$ is any (necessarily continuous) function from $X$ to a compact Hausdorff space $Y$, then $\phi$ has a continuous extension $\phi^{\beta}: \beta X \rightarrow Y$. For more details of this construction, see [11, $\$ 7$ or 7, Chapter 6].

Given a discrete topological semigroup $(S,+)$, we extend the operation + to $\beta S$ in steps. First, for $s \in S$, the function $\rho_{s}$ defined by

$$
\rho_{s}(t)=t+s, \quad t \in S,
$$

takes $S$ to $\beta S$. For $p \in \beta S \backslash S$ and $s \in S$, let $p+s=\rho_{s}^{\beta}(p)$; thus " + " is defined on $\beta S \times S$. For $p \in \beta S$, the function $\lambda_{p}$ defined by

$$
\lambda_{p}(t)=p+t, \quad t \in S,
$$

takes $S$ to $\beta S$, and therefore has a continuous extension $\lambda_{p}^{\beta}: \beta S \rightarrow \beta S$. Hence, for $p$, $q \in \beta S \backslash S$, we may define

$$
p+q=\lambda_{p}^{\beta}(q) \text {. }
$$

Now " + " denotes a left continuous binary operation on $\beta S$. (In a similar manner, one could extend " + " on $S$ as a right continuous binary operation on $\beta S$; we make no use of that construction in this paper.) 
For $s \in S$ and $A \subseteq S$, define

$$
A-s=\{t \in S \mid t+s \in A\} .
$$

2.1 Lemma. For $(S,+)$, a discrete topological semigroup, let $p \in \beta S$, let $s \in S$ and let $A \subseteq S$. Then $A \in p+s$ if and only if $A-s \in p$.

Proof. Assume first that $A \in p+s$, and pick a basic neighborhood $\bar{B}$ of $p$ such that $\rho_{s}^{\beta}[\bar{B}] \subseteq \bar{A}$. In particular, then $\rho_{s}[B] \subseteq A$, so that (everything is happening in $S$ now) $B \subseteq A-s$. Thus $A-s \in p$, since $p$ is a filter.

Now suppose that $A-s \in p$, and suppose that $A \notin p+s$. Then $S \backslash A \in p+s$, since $p+s$ is an ultrafilter. But then, by the above, $(S \backslash A)-s \in p$, while

$$
((S \backslash A)-s) \cap(A-s)=\varnothing \text {. }
$$

It is well known that the Stone-Čech compactification $\beta S$ of a discrete semigroup $S$ can be given a left topological semigroup structure [3, III.14.5(i), p. 126]. In the following theorem we give an explicit construction of that left topological semigroup structure on the space of ultrafilters $\beta S$. This provides a departure point for consideration in $\$ \S 5$ and 6 of semitopological semigroup structures on spaces of filters.

2.2 TheOREM. Let $(S,+)$ be a discrete topological semigroup. Then $(\beta S,+)$ is a left topological semigroup, and for each $s \in S$, the function $p \rightarrow p+s$ from $\beta S$ to $\beta S$ is continuous.

Proof. We have already established everything except the associativity of + on $\beta S$. To this end, let $p, q$ and $t$ be in $\beta S$, and suppose that

$$
p+(q+t) \neq(p+q)+t \text {. }
$$

Pick $A \subseteq S$ such that $A \in p+(q+t)$ and $A \notin(p+q)+t$. Pick $B \in q+t$ such that $A \in p+r$ whenever $B \in r$. Then pick $C \in t$ such that $S \backslash A \in(p+q)+r$ whenever $C \in r$; and, finally, pick $D \in t$ such that $B \in q+r$ whenever $D \in r$. Then $C \cap D \in t$, so choose $s \in C \cap D$. Then $C \in \hat{s}$, so $S \backslash A \in(p+q)+s$. Also $D \in \hat{s}$, so $B \in q+s$. Thus by Lemma 2.1, $(S \backslash A)-s \in p+q$ and $B-s \in q$. Pick $E \in q$ so that $(S \backslash A)-s \in p+r$ whenever $E \in r$, and choose $k \in(B-s) \cap E$. Then $E \in \hat{k}$, so $(S \backslash A)-s \in p+k$, and, by Lemma $2.1,((S \backslash A)-s)-k \in p$. Since $k \in B-s$, we have $k+s \in B$, so $A \in p+(k+s)$. Thus, by Lemma $2.1, A-(k$ $+s) \in p$. But

$$
(((S \backslash A)-s)-k) \cap(A-(k+s))=\varnothing
$$

since + is associative on $S$, and we have the contradiction.

For any space $X$, with $C(X)$ the set of bounded continuous complex-valued functions on $X$ with the sup-norm topology, we let $C(X)^{*}$ be the set of bounded linear functionals from $C(X)$ to $\mathbf{C}$. The topology of pointwise covergence, or simply the pointwise topology, on $C(X)$ is the topology inherited by $C(X)$ as a subspace of the produce space $\mathbf{C}^{X}$. The weak topology $\sigma\left(C(X), C(X)^{*}\right)$ on $C(X)$ is the coarsest topology on $C(X)$ such that each member of $C(X)^{*}$ is continuous. Likewise, the weak* $^{*}$ topology $\sigma\left(C(X)^{*}, C(X)\right)$ on $C(X)^{*}$ is the coarsest topology on $C(X)^{*}$ such that each member of $C(X)$, considered as a subset of $C(X)^{* *}$, is continuous. 
A mean on $C(X)$ is a bounded linear functional $\mu \in C(X)^{*}$ such that $\|\mu\|=1$. (The norm in $C(X)^{*}$ is the sup norm over the unit ball in $C(X)$.) By the Alaoglu theorem, the unit ball in $C(X)^{*}$ is compact (and Hausdorff). For each $x \in X$, define a mean $e(x)$ by

$$
e(x)(f)=f(x), \quad f \in C(X) .
$$

The map $x \rightarrow e(x)$ from $X$ to $C(X)^{*}$ is continuous when $C(X)^{*}$ has the weak* topology [3, Proposition I.3.5, p. 14], but if $X$ is not completely regular it may not be one-to-one. Define $\beta X$ to be the weak ${ }^{*}$ closure of $\{e(x) \mid x \in X\}$ in $C(X)^{*}$. Then $\beta X$ is a compact Hausdorff space with the property that if $\phi$ is a continuous function from $X$ to a compact Hausdorff space $Y$ then there is an "extension" $\phi^{\beta}$ of $\phi$ to $\beta X$ in the sense that

$$
\phi^{\beta}(e(x))=\phi(x), \quad x \in X .
$$

If $X$ is completely regular, then $\beta X$ is, up to homeomorphism, the Stone-Čech compactification of $X$.

Let $(S,+)$ be a left topological semigroup. For $s \in S$ and $f \in C(S)$, define the function $f_{s}$ by

$$
f_{s}(t)=f(s+t), \quad t \in S .
$$

(Thus, $f_{s}=f \circ \lambda_{s}$, and hence $f_{s} \in C(S)$.) Recall that a function $f \in C(S)$ is weak almost periodic if

$$
\lim _{n \rightarrow \infty} \lim _{k \rightarrow \infty} f\left(t_{n}+s_{k}\right)=\lim _{k \rightarrow \infty} \lim _{n \rightarrow \infty} f\left(t_{n}+s_{k}\right),
$$

whenever $\left\langle t_{n}\right\rangle_{n=1}^{\infty}$ and $\left\langle s_{k}\right\rangle_{k=1}^{\infty}$ are sequences in $S$ and all limits involved exist. $W(S)$ denotes the set of weak almost periodic functions.

2.3 THEOREM. Let $S$ be a semitopological semigroup, and let $f \in C(S)$. The following statements are equivalent.

(a) $f \in W(S)$.

(b) $\operatorname{cl}\left\{f_{s} \mid s \in S\right\}$ is compact in $C(S)$ with the weak topology.

(c) $\operatorname{cl}\left\{f_{s}^{\beta} \mid s \in S\right\}$ is compact in $C(\beta S)$ with the pointwise topology.

(d) If $\left\langle s_{n}\right\rangle_{n=1}^{\infty}$ is a sequence in $S$, then there is a subsequence $\left\langle t_{k}\right\rangle_{k=1}^{\infty}$ of $\left\langle s_{n}\right\rangle_{n=1}^{\infty}$ such that the sequence $\left\langle f_{t_{k}}^{\beta}\right\rangle_{k=1}^{\infty}$ converges in $C(\beta S)$ with the pointwise topology.

PRoof. Statements (a) and (b) are, respectively, statements (c) and (a) of Theorem III.8.2, p. 108, of [3], and are shown there to be equivalent. Statements (b), (c) and (d) are, respectively, statements (a), (b) and (d) of Theorem I.1.8, p. 17, of [2] with $K=\left\{f_{s}: s \in S\right\}$ and are shown there to be equivalent.

2.4 THEOREM. Let $S$ be a semitopological semigroup. There is a compact semigroup $\delta S$ and a continuous homomorphism $\delta_{S}: S \rightarrow \delta S$ such that

(1) $\delta S$ is a left topological semigroup,

(2) $\delta_{S}[S]$ is dense in $\delta S$,

(3) for every $s \in S$, the function

$$
t \rightarrow t+\delta_{S}(s): \delta S \rightarrow \delta S
$$

is continuous, and 
(4) the pair $\left(\delta S, \delta_{S}\right)$ is maximal with respect to these properties in the sense that if $\phi$ is a continuous homomorphism from $S$ to a compact semigroup $T$ and $(T, \phi)$ satisfies (1), (2) and (3) with $\phi$ replacing $\delta_{S}$ and $T$ replacing $\delta S$, then there is a continuous homomorphism $\eta$ from $\delta S$ onto $T$ such that $\eta \circ \delta_{S}=\phi$.

If $S$ is discrete, then $\left(\delta S, \delta_{S}\right)$ is, up to isomorphism, the Stone-Čech compactification with semigroup structure as given in Theorem 2.2 .

Proof. This is the $\Re \Re \mathcal{R}$-compactification of $S$ opposite to the $\mathcal{Q} \mathbb{Q}$-compactification of [3, Theorem III.4.5, p. 104]. The assertion about discrete semigroups follows from (4) and the familiar universal mapping property of the Stone-Čech compactification. (See also [3, Remark III.14.5(i), p. 126].)

Even though we are writing our semigroups additively, we shall use the standard semigroup term for a "zero" of a semigroup. That is, $z$ is a zero of $(S,+)$ if and only if

$$
z+x=x+z=z, \quad \forall x \in S .
$$

(Thus, the number 0 is not a zero of $(\mathbf{Z},+)$ but an identity.)

Comment. If $S$ is not discrete, then $\delta S$ need not equal $\beta S$, even if $S$ is completely regular. Example [3, V.2.3(b), p. 176] is such a semigroup.

2.5 THEOREM. Let $S$ be a semitopological semigroup. There is a compact semigroup $\omega S$ and a continuous homomorphism $\omega_{S}: S \rightarrow \omega S$ such that

(1) $\omega S$ is a semitopological semigroup,

(2) $\omega_{S}[S]$ is dense in $\omega S$, and

(3) the pair $\left(\omega S, \omega_{S}\right)$ is maximal with respect to these properties in the sense that if $\phi$ is a continuous homomorphism from $S$ to a compact semigroup $T$, and $(T, \phi)$ satisfies (1) and (2) with $\phi$ replacing $\omega_{S}$ and $T$ replacing $\omega S$, then there is a continuous homomorphism $\eta$ from $\omega S$ onto $T$ such that $\eta \circ \omega_{S}=\phi$. Moreover,

(4) a function $f \in C(S)$ extends to $\omega S$ (i.e., there is a function $f^{\omega} \in C(\omega S)$ with $\left.f=f^{\omega} \circ \omega_{S}\right)$ if and only if $f$ is weak almost periodic. Also,

(5) if $S$ is abelian, so is $\omega S$.

Proof. This is [3, Theorems III.8.4 and III.8.3], except statement (5) which follows from the fact that $\omega_{S}[S]$ is a dense commutative subset and [2, Lemma II.3.1 (iv)].

The pair $\left(\omega S, \omega_{S}\right)$ is called the weak almost periodic compactification of $S$. Sometimes it really is a compactification in the sense of topology.

2.6 THEOREM. Let $S$ be a locally compact (and noncompact) semitopological semigroup. If the following equivalent conditions hold, then $\left(\omega S, \omega_{S}\right)$ is a topological compactification of $S$. (That is, $\omega_{S}$ is a homeomorphism.)

(a) The one-point compactification of $S$ is a semitopological semigroup with the point at infinity acting as a zero.

(b) The space $C_{0}(S)$ of continuous complex-valued functions vanishing at infinity is invariant under right and left translations by elements of $S$.

(c) If $s \in S$ and $K$ is a compact subset of $S$, then there is a compact set $K^{\prime} \subseteq S$ such that if $x \in S \backslash K^{\prime}$, then $s+x \notin K$ and $x+s \notin K$.

(d) The function $\delta_{S}: S \rightarrow \delta_{S}[S]$ of Theorem 2.4 is an isomorphism of left topological semigroups and $\delta S \backslash \delta_{S}[S]$ is a closed two-sided ideal of $\delta S$. 
Proof. Statements (a), (b) and (c) are, respectively, statements (b), (a) and (b') of [2, Theorem III.4.6, p. 133], which are shown there to be equivalent and to imply that $\omega_{S}$ is a homeomorphism. (It should be noted, however, that the phrase "and $J$ is not empty" should be added to Lemma III.4.1(b) and Proposition III.4.5(c) of [2] to make the proof of Theorem III.4.6 valid.)

To see that (a) implies (d), note that $(S \cup\{\infty\}, i)$ satisfies conditions (1), (2) and (3) of Theorem 2.4, where $i(s)=s$. (That condition (3) holds follows from statement (c).) Pick a continuous homomorphism, by way of (4) of Theorem 2.4,

$$
\eta: \delta S \rightarrow S \cup\{\infty\}
$$

such that $\eta \circ \delta_{S}=i$. Thus $\delta_{S}$ is one-to-one and the restriction of $\eta$ to $\delta_{S}[S]$ is $\eta \circ \delta_{S} \circ \delta_{S}^{-1}=i \circ \delta_{S}^{-1}=\delta_{S}^{-1}$, which implies that $\delta_{S}$ is a homeomorphism and an isomorphism. Also $\delta_{S} \circ \eta$ is continuous and is equal to the identity on $\delta_{S}[S]$ and, hence, is the identity on its domain. Thus its domain is $\delta_{S}[S]$ so that $\eta^{-1}[\{\infty\}]=$ $\delta S \backslash \delta_{S}[S]$. That $\delta S \backslash \delta_{S}[S]$ is a closed ideal now follows easily.

To see that (d) implies (a), note that the quotient of $\delta S$ obtained by collapsing that closed ideal $\delta S \backslash \delta_{S}[S]$ to a point is the one-point compactification of $S$ with the point at infinity acting as a zero.

It is easy to produce examples of semigroups which fail to satisfy the conditions of Theorem 2.6- any infinite discrete semigroup with a zero element, such as $(\mathbf{Z}, \cdot)$, will do. We shall determine in $\S 6$ exactly when $\omega S$ is a topological compactification of $S$ for discrete $S$. (For an example of a nonlocally compact semigroup $S$ for which $\omega S$ is not a topological compactification, take $S$ to be a semitopological nontopological group, such as the additive real numbers with the topology generated by half-open intervals $[a, b)$. By [14, Corollary 6.3, p. 282] a subgroup of a compact semitopological semigroup must be a topological group.)

The conditions of Theorem 2.6 do hold in the following important cases.

(1) $S$ is a locally compact, noncompact, topological group. (In this case condition (c) is easily verified-let $K^{\prime}=\rho_{-s}[K] \cup \lambda_{-s}[K]$.)

(2) $S$ is any closed subsemigroup of a locally compact, noncompact, topological group $G$. (Again we verify conditon (c). Let $K$ be a compact subset of $S$ and let $s \in S$. Then $K$ is a compact subset of $G$, so pick a compact $K^{\prime} \subseteq G$ such that $x+s \notin K$ and $s+x \notin K$ whenever $x \in G \backslash K^{\prime}$. Let $K^{\prime \prime}=S \cap K^{\prime}$. Then $K^{\prime \prime}$ is compact since $S$ is closed, and if $x \in S \backslash K^{\prime \prime}$, then $x \in G \backslash K^{\prime}$, and the result follows.)

Let $\mathscr{F}$ be a vector subspace of $C(S)$. If $f \in \mathscr{F}$ implies that $f_{s} \in \mathscr{F}$ for every $s \in S$, then $\mathscr{F}$ is called left-invariant. A mean $\mu \in \mathscr{F}^{*}$ is called left-invariant if

$$
\mu\left(f_{s}\right)=\mu(f), \quad \forall s \in S, \forall f \in \mathscr{F} .
$$

One defines right-invariance similarly.

A right ideal in a semigroup $S$ is a nonempty subset $J \subseteq S$ such that

$$
J+s \subseteq J, \quad \forall s \in S .
$$

A minimal right ideal of $S$ is a right ideal which does not contain properly any right ideal of $S$. 
Given $f \in C(S)$, the right orbit is defined by

$$
O(f)=\left\{f \circ \rho_{s} \mid s \in S\right\}
$$

For $s \in S$ define the continuous linear operator $R_{s}$ by

$$
R_{s} f=f \circ \rho_{s}, \quad \forall f \in C(S) .
$$

The following theorem shows the connection between left-invariant means and minimal right ideals.

2.7 THEOREM. Let $S$ be a semitopological semigroup with identity. Then $\omega S$ contains at least one minimal right ideal, and the following statements are equivalent.

(a) $\omega S$ contains only one minimal right ideal.

(b) $W(S)$ has a left-invariant mean.

(c) The closed convex hull $\overline{c o}(O(f))$ of the right orbit of $f$ contains a constant function.

Proof. One way to obtain $\omega S$ is as the set of multiplicative means $\mathfrak{R} \mathscr{T}(W(S))$ on $W(S)[3$, III.2.2, p. 98 and III.8.3, p. 110]. With that observation we get, from [3, Theorem II.5.6, p. 82], the equivalence of (a) and (b) and the equivalence of (a) and

$\left(\mathrm{a}^{\prime}\right) \mathfrak{R}(W(S))$ contains only one minimal right ideal, where $\mathfrak{R}(W(S))$ $=\overline{\operatorname{co}}(\mathfrak{N} \mathscr{N}(W(S)))$ is the set of means on $W(S)$.

Another way to obtain $\omega S$ is as the weak operator closure $T_{W}$ of the set of operators $\left\{R_{s} \mid s \in S\right\}$ on $W(S)$ [2, III.2.2, 2.4 and 2.11, pp. 120-126]. Under that identification $\mathscr{R}(W(S))$ can be identified with the (weak operator) closed convex hull $T_{W}^{\prime}$ of $T_{W}[2$, p. 81; 3, Proposition 3.5, p. 14 and Proposition 3.9, p. 16]. Now (a') is equivalent to

(a") $T_{W}^{\prime}$ contains only one minimal right ideal, which by [2, II. 3.19, p. 76] is equivalent to

(c') $T_{W}^{\prime} f$ contains a fixed point of $T$ for every $f \in W(S)$. But $T_{W}^{\prime} f=\overline{\operatorname{co}}(O(f))$ and a fixed point under a right translation operator is a constant function.

(With a different viewpoint, this theorem could also be derived from [3, Theorem II.1.38, p. 48].)

The next two lemmas, whose straightforward proofs are omitted, are stated here for later reference.

2.8 LemMA. Let $S$ be a semitopological semigroup, and let $f, g \in W(S)$ be such that $\operatorname{Range}(f) \subseteq \mathbf{R}$ and $\operatorname{Range}(g) \subseteq \mathbf{R}$. Define $h$ by

$$
h(s)=\max \{f(s), g(s)\}, \quad s \in S \text {. }
$$

Then $h \in W(S)$.

2.9 LemMA. Let $S$ be a compact semitopological semigroup. Then $W(S)=C(S)$.

3. The weak almost periodic compactification of a semitopological semigroup. We recall from Theorem 2.5(5) that if $S$ is abelian, so is $\omega S$. The following theorem characterizes weak almost periodic functions in terms of the (noncommutative) maximal left topological compactification $\left(\delta S, \delta_{S}\right)$ of Theorem 2.4. 
3.1 THEOREM. Let $S$ be an abelian semitopological semigroup, and let $f \in C(S)$. Then $f \in W(S)$ if and only if there is a function $f^{\delta} \in C(\delta S)$ such that $f=f^{\delta} \circ \delta_{S}$ and

$$
f^{\delta}(p+q)=f^{\delta}(q+p), \quad \forall p, q \in \delta S .
$$

Proof. (Necessity) By Theorem 2.5(4), if $f \in W(S)$, then there is a function $f^{\omega} \in C(\omega S)$ such that $f=f^{\omega} \circ \omega_{S}$. Since $\left(\omega S, \omega_{S}\right)$ satisfies conditions (1), (2) and (3) of Theorem 2.4, there is a continuous homomorphism $\eta: \delta S \rightarrow \omega S$ such that $\eta \circ \delta_{s}=\omega_{s}$.

Consider the continuous function

$$
f^{\omega} \circ \eta: \delta S \rightarrow \mathbf{C} .
$$

Since $\left(f^{\omega} \circ \eta\right) \circ \delta_{S}=f^{\omega} \circ\left(\eta \circ \delta_{S}\right)=f^{\omega} \circ \omega_{S}=f$, we have that $f^{\delta}=f^{\omega} \circ \eta$ is such that $f=f^{\delta} \circ \delta_{S}$. Now let $p, q \in \delta S$. Then since $\eta$ is a homomorphism and $\omega S$ is abelian,

$$
\begin{aligned}
f^{\delta}(p+q) & =f^{\omega}(\eta(p+q))=f^{\omega}(\eta(p)+\eta(q)) \\
& =f^{\omega}(\eta(q)+\eta(p))=f^{\omega}(\eta(q+p))=f^{\delta}(q+p) .
\end{aligned}
$$

(Sufficiency) Let $\left\langle t_{n}\right\rangle_{n=1}^{\infty}$ and $\left\langle s_{k}\right\rangle_{k=1}^{\infty}$ be sequences in $S$ such that all of the following limits exist:

(1) for all $n \in \mathbf{N}, a_{n}=\lim _{k \rightarrow \infty} f\left(t_{n}+s_{k}\right)$,

(2) for all $k \in \mathbf{N}, b_{k}=\lim _{n \rightarrow \infty} f\left(t_{n}+s_{k}\right)$,

(3) $\lim _{n \rightarrow \infty} a_{n}=a$, and

(4) $\lim _{k \rightarrow \infty} b_{k}=b$.

By the definition preceding Theorem 2.3, we must show that $a=b$.

Pick cluster points $p$ and $q$ in $\delta S$ of the sequences $\left\langle\delta_{S}\left(t_{n}\right)\right\rangle_{n=1}^{\infty}$ and $\left\langle\delta_{S}\left(s_{k}\right)\right\rangle_{k=1}^{\infty}$, respectively. Note that for each $k, p+\delta_{S}\left(s_{k}\right)$ is a cluster point of

$$
\left\langle\delta_{S}\left(t_{n}\right)+\delta_{S}\left(s_{k}\right)\right\rangle_{n=1}^{\infty}
$$

by Theorem 2.4(3); for each $n, \delta_{S}\left(t_{n}\right)+q$ is a cluster point of

$$
\left\langle\delta_{S}\left(t_{n}\right)+\delta_{S}\left(s_{k}\right)\right\rangle_{k=1}^{\infty},
$$

$p+q$ is a cluster point of $\left\langle p+\delta_{S}\left(s_{k}\right)\right\rangle_{k=1}^{\infty}$, and $q+p$ is a cluster point of

$$
\left\langle q+\delta_{S}\left(t_{n}\right)\right\rangle_{n=1}^{\infty}
$$

Thus, for each $n$,

$$
\begin{aligned}
a_{n} & =\lim _{k \rightarrow \infty} f\left(t_{n}+s_{k}\right)=\lim _{k \rightarrow \infty} f^{\delta}\left(\delta_{S}\left(t_{n}+s_{k}\right)\right) \\
& =\lim _{k \rightarrow \infty} f^{\delta}\left(\delta_{S}\left(t_{n}\right)+\delta_{S}\left(s_{k}\right)\right)=f^{\delta}\left(\delta_{S}\left(t_{n}\right)+q\right) .
\end{aligned}
$$

Likewise, $b_{k}=f^{\delta}\left(p+\delta_{S}\left(s_{k}\right)\right)$ and $b=f^{\delta}(p+q)$. Now by the hypothesis about $f^{\delta}$, we get

$$
a_{n}=f^{\delta}\left(\delta_{S}\left(t_{n}\right)+q\right)=f^{\delta}\left(q+\delta_{S}\left(t_{n}\right)\right)
$$

so

$$
a=\lim _{n \rightarrow \infty} a_{n}=\lim _{n \rightarrow \infty} f^{\delta}\left(q+\delta_{S}\left(t_{n}\right)\right)=f^{\delta}(q+p)=f^{\delta}(p+q),
$$

and we have that $a=f^{\delta}(p+q)=b$, as desired. 
3.2 COROLlaRY. Let $S$ be a discrete abelian topological semigroup, and let $f \in C(S)$. Then $f \in W(S)$ if and only if

$$
f^{\beta}(p+q)=f^{\beta}(q+p)
$$

for all $p$ and $q$ in $\beta S$.

We now turn our attention to subsemigroups $S$ of locally compact topological groups $G$.

3.3 Comment. If $S$ is a discrete semigroup, then addition is clearly jointly continuous at every point of $S \times \omega S$. This is also true under various other hypotheses, including $S$ being a locally compact group. (See, for example, [13 and 14].) Is it always true for closed subsemigroups of locally compact groups?

3.4 THEOREM. Let $(G,+)$ be a locally compact topological group, and let $S$ be a closed, noncompact subsemigroup of $G$. Suppose that $W(S)$ has an invariant mean (left- and right-) and that addition is continuous at every point of $S \times \omega S$. Then there is a closed translation invariant ideal $W(S)_{0}$ such that

$$
W(S)=W(S)_{0} \oplus W(S)_{p},
$$

where $W(S)_{p}$ is the set of strong almost periodic functions on $S$. Moreover, the following statements about a function $f \in W(S)$ are equivalent.

(a) $f \in W(S)_{0}$.

(b) $f^{\omega} \equiv 0$ on the minimal ideal $M(\omega S)$ of $\omega S$.

(c) $\mu(|f|)=0$, where $\mu$ is the unique invariant mean on $W(S)$.

(d) The zero function is in the weak closure of the right orbit $O(f)$.

(e) For every $\varepsilon>0$ and every compact subset $K$ of $S$, there is an element $s \in S$ such that

$$
\varepsilon>\left\|R_{s} f\right\|_{K}=\sup \{|f(k+s)|: k \in K\} .
$$

Proof. That, in the presence of an invariant mean, $W(S)$ decomposes into the direct sum $W(S)_{0} \oplus W(S)_{p}$ is well known, as is the equivalence of (a)-(d). (See, for example, [4, pp. 29-30].)

For the implication (b) $\Rightarrow(e)$, we establish the contrapositive. So suppose that for some compact $K$ and some $\varepsilon>0$, there does not exist $s \in S$ such that $\left\|R_{s} f\right\|_{K}<\varepsilon$. Since $W(S)$ has an invariant mean, the minimal ideal $M(\omega S)$ is a compact topological group [15, Theorem 5.7, p. 85]. Let $\left\langle s_{\alpha}\right\rangle_{\alpha \in D}$ be a net in $S$ such that

$$
e=\lim _{\alpha \in D} \omega_{S}\left(s_{\alpha}\right)
$$

where $e$ is the identity of the group $M(\omega S)$. Now for each $\alpha \in D$, there is an element $k_{\alpha} \in K$ with

$$
\left|f\left(k_{\alpha}+s_{\alpha}\right)\right| \geqslant \varepsilon .
$$

Passing to a subnet if necessary, we may assume that $\left\langle k_{\alpha}\right\rangle_{\alpha \in D}$ converges, say to $k$. Since addition is continuous on $S \times \omega S$, we get that

$$
\lim _{\alpha \in D} \omega_{S}\left(k_{\alpha}+s_{\alpha}\right)=\lim _{\alpha \in D}\left[\omega_{S}\left(k_{\alpha}\right)+\omega_{S}\left(s_{\alpha}\right)\right]=\omega_{S}(k)+e .
$$


Since $M(\omega S)$ is an ideal, $\omega_{S}(k)+e \in M(\omega S)$. But

$$
\begin{aligned}
\varepsilon & \leqslant\left|\lim _{\alpha \in D} f\left(k_{\alpha}+s_{\alpha}\right)\right|=\left|\lim _{\alpha \in D} f^{\omega}\left(\omega_{S}\left(k_{\alpha}+s_{\alpha}\right)\right)\right| \\
& =\left|f^{\omega}\left(\lim _{\alpha \in D} \omega_{S}\left(k_{\alpha}+s_{\alpha}\right)\right)\right|=\left|f^{\omega}\left(\omega_{S}(k)+e\right)\right|,
\end{aligned}
$$

so $f^{\omega}\left(\omega_{S}(k)+e\right) \neq 0$ and we have established the contrapositive.

Now we show that (e) implies (d). Let $\mathscr{K}$ be the set of all compact subsets of $S$ directed by $\subseteq$. Fix $\varepsilon>0$. For each $\alpha \in \mathscr{K}$, there is an element $s_{\alpha} \in S$ with

$$
\left|f\left(k+s_{\alpha}\right)\right|<\varepsilon \text { for every } k \in \alpha .
$$

Every $t \in S$ is in some compact set $\alpha_{0} \in \mathscr{K}$; and if $\alpha_{0} \subseteq \alpha$, then $t \in \alpha$, so

$$
\left|f\left(t+s_{\alpha}\right)\right|<\varepsilon, \quad \forall \alpha \supseteq \alpha_{0} .
$$

Thus, if $g_{1}$ is a weak cluster point of the net $\left\langle R_{s_{\alpha}} f\right\rangle_{\alpha \in \mathscr{K}}$, we must have that

$$
\left|g_{1}(t)\right|<\varepsilon \text { for all } t \in S \text {. }
$$

In like manner, obtain $g_{n} \in \operatorname{cl}(O(f))$ with

$$
\left|g_{n}(t)\right|<\varepsilon / n \text { for all } t \in S \text {. }
$$

The sequence $\left\langle g_{n}\right\rangle_{n=1}^{\infty}$ must converge weakly to 0 because zero is its only pointwise cluster point, and we have that zero is in the weak closure of $O(f)$, as desired.

3.5 LemMa. With $S$ as in Theorem 3.4, the function

$$
S \rightarrow L_{s} f: S \rightarrow W(S)
$$

is continuous, where $L_{s} f(t)=f(s+t)$, when $W(S)$ has the sup-norm topology. As a result, functions in $W(S)$ are uniformly continuous on $S$.

Proof. The addition function

$$
(s, p) \rightarrow \omega_{S}(s)+p: S \times \omega S \rightarrow \omega S
$$

is continuous. By [3, Theorem III.11.3, p. 121], $\omega S$ is the $2 \mathcal{Q}(5)$-compactification of $S$; which means, by [3, Definition III.11.1, p. 121 and Definition III.5.1, p. 104], that the function $s \rightarrow L_{s} f: S \rightarrow C(S)$ is norm continuous. In other words, if $\varepsilon>0, s_{0} \in S$ and

$$
U=\left\{g \in C(S) \mid\left(\sup _{t \in S}\left|g(t)-f\left(s_{0}+t\right)\right|\right)<\varepsilon\right\}
$$

then there is a compact neighborhood of 0 in $G$ such that if $v \in V$, then $L_{v+s_{0}} f \in U$; that is, for every $t \in S$,

$$
\left|L_{v+s_{0}} f(t)-f_{s_{0}}(t)\right|=\left|f\left(v+s_{0}+t\right)-f\left(s_{0}+t\right)\right|<\varepsilon .
$$

The last condition implies that $f$ is uniformly continuous with respect to the right uniform structure on $G$ relativized to $S$.

There is a corresponding other hand theorem; so $f$ is uniformly continuous no matter which uniform structure is being considered.

Theorem 3.4 says that there are arbitrarily large "dips below $\varepsilon$ " in a function $f \in W(S)_{0}$. The following theorem says, on the other hand, that "rises above $\varepsilon$ " between dips of a specified size are of a uniformly bounded size. 
3.6 THEOREM. Let $(G,+)$ be a locally compact topological group, and let $S$ be a closed, noncompact, subsemigroup of $G$ containing the identity 0 . Suppose that $W(S)$ has an invariant mean and that addition is continuous at every point of $S \times \omega S$. Suppose $f \in W(S)_{0}$. Let $U_{0}$ be a compact neighborhood of the identity 0 of $G$, and let $\varepsilon>0$. Then there is a compact neighborhood $V=V\left(U_{0}, f, \varepsilon\right)$ of 0 in $G$ such that, for every $s \in S$, there exists $r \in S$ such that

$$
(V+s) \cap\left(U_{0} \dot{\cap} S+r\right) \neq \varnothing
$$

and

$$
\varepsilon>\left\|R_{r} f\right\|_{U_{0}}=\sup \left\{|f(t+r)|: t \in U_{0} \cap S\right\} .
$$

(Loosely speaking, this says that no matter where $V$ is placed in $S$, it will intersect a set as big as $U_{0}$ where $|f|$ dips below $\varepsilon$.)

Proof. Define

$$
C=\left\{x \in S \mid \exists y \in S \text { with } x \in U_{0}+y \text { and }\left\|R_{y} f\right\|_{U_{0}}<\varepsilon\right\} .
$$

( $C$ is the union of all the sets at least the size of $U_{0}$ where $|f|$ dips below $\varepsilon$.) By Theorem 3.4, $C$ is not empty since $U_{0} \cap S$ is compact. The theorem states that there is a $V$ such that for every $s \in S,(V+s) \cap C \neq \varnothing$. Suppose, by way of contradiction that there is no such $V$. Let $\mathcal{Q}$ be the family of compact neighborhoods of 0 in $G$ directed by $\subseteq$. (Note that we have taken the reverse order to the usual one on $U_{\text {.) }}$ For each $V \in$ Q , there is some $s_{V} \in S$ such that

$$
\left(V+s_{V}\right) \cap C=\varnothing .
$$

Now $\left\langle R_{s_{V}} f\right\rangle_{V \in \mathcal{Q}}$ is a net in the weak compact set $\operatorname{cl}(O(f))$; so passing to a subnet if necessary, we may assume that $\left\langle R_{s_{V}} f\right\rangle_{V \in \mathcal{U}}$ converges to, say, $f_{0} \in \operatorname{cl}(O(f))$. Since $W(S)_{0}$ is closed [15, Theorem 4.9(iv), p. 78], it is weakly closed [6, Theorem V.3.13, p. 422]; hence, $f_{0} \in W(S)_{0}$.

Let $K=U_{0} \cap S$. Since $K$ is compact, by Theorem 3.4 there is an element $s_{0} \in S$ such that

$$
\varepsilon / 3>\sup \left\{\left|f_{0}\left(u+s_{0}\right)\right|: u \in K\right\} .
$$

Let $t=t_{0} \in K+s_{0}$. Since the weak limit of $\left\langle R_{s_{V}} f\right\rangle_{V \in \mathcal{Q}}$ is $f_{0}$, the pointwise limit of the net is also $f_{0}$. Thus, there is some $V_{0} \in \mathcal{Q}$ such that if $V \supseteq V_{0}$, then

$$
\left|f\left(t_{0}+s_{V}\right)-f_{0}\left(t_{0}\right)\right|<\varepsilon / 3 .
$$

By Lemma 3.5, the function $s \rightarrow L_{s} f: S \rightarrow W(S)$ is continuous, so there is some compact neighborhood $W=W_{0}$ of 0 in $G$ such that

$$
\left|f\left(w+t_{0}+s\right)-f\left(t_{0}+s\right)\right|<\varepsilon / 3
$$

for all $s \in S$ and $w \in W_{0}$ with $w+t_{0} \in S$. Putting all this together, we have that for every $V \supseteq V_{0}$ and every $w \in W_{0}$ with $w+t_{0} \in S$,

$$
\begin{aligned}
\left|f\left(w+t_{0}+s_{V}\right)\right| \leqslant & \left|f\left(w+t_{0}+s_{V}\right)-f\left(t_{0}+s_{V}\right)\right| \\
& +\left|f\left(t_{0}+s_{V}\right)-f_{0}\left(t_{0}\right)\right|+\left|f_{0}\left(t_{0}\right)\right| \\
< & </ 3+\varepsilon / 3+\varepsilon / 3 .
\end{aligned}
$$


For each $t \in K+s_{0}$ obtain a $W$ as above; cover the compact set $K+s_{0}$ with the $(W+t)$ 's and extract a finite subcover $\left\{W_{1}+t_{1}, W_{2}+t_{2}, \ldots, W_{n}+t_{n}\right\}$. Let $\left\{V_{1}, V_{2}, \ldots, V_{n}\right\}$ be the corresponding $V$ 's. Now if $V \in \mathcal{U}$ is such that

$$
V \supseteq V_{1} \cup \cdots \cup V_{n} \text {, }
$$

then

$$
\varepsilon>\sup \left\{\left|f\left(z+s_{V}\right)\right|: z \in\left(W_{k}+t_{k}\right) \cap S\right\}
$$

in particular, if $x \in K+s_{0}$, then

$$
\varepsilon>\left|f\left(x+s_{V}\right)\right| \text {. }
$$

Thus for any $x \in K+s_{0}$ and any $V \supseteq V_{1} \cup \cdots \cup V_{n}, x+s_{V} \in C$. But eventually, there is a $V \supseteq V_{1} \cup \cdots \cup V_{n}$ with $V \cap K \neq \varnothing$, in which case there is an element $x$ with

$$
x+s_{V} \in\left(V+s_{V}\right) \cap C,
$$

contradicting the defining assumption on the net $\left\langle s_{V}\right\rangle_{V \in \mathcal{Q}}$.

3.7 Corollary. Let $S$ be as in Theorem 3.6. Suppose $f \in C(S)$ is real valued. For real numbers $a$ and $\delta, \delta>0$, define

$$
A=A(a, \delta)=\{s \in S \mid f(s)<a-\delta\}
$$

and

$$
B=B(a, \delta)=\{s \in S \mid f(s)>a+\delta\}
$$

if for any compact $K \subseteq S$ it is possible to find $t_{1} \in S$ and $t_{2} \in S$ such that

$$
K+t_{1} \subseteq A \text { and } K+t_{2} \subseteq B \text {, }
$$

then $f \notin W(S)$.

Proof. Suppose $A$ and $B$ satisfy the stated conditions, but that $f \in W(S)$. Let

$$
g(s)=\max \{f(s)-a+\delta, 0\} .
$$

By Lemma 2.8, $g \in W(S)$. Let $\varepsilon=2 \delta>0$. Then

$$
A=\{s \in S \mid g(s)=0\} \quad \text { and } \quad B=\{s \in S \mid g(s)>\varepsilon\} .
$$

(Note that $g(s) \geqslant 0$ for all $s \in S$.) By Theorem 3.4(e), $g \in W(S)_{0}$ since $A$ contains arbitrarily large blocks. Fix a compact neighborhood $U_{0}$ of 0 in $G$. By Theorem 3.6, there is a compact neighborhood $V$ of 0 in $G$ such that, for every $s \in S$, there exists $r \in S$ such that

$$
(V+s) \cap\left(U_{0}+r\right) \cap S=\varnothing
$$

and

$$
\sup \left\{|g(t)|: t \in\left(U_{0}+r\right) \cap S\right\}<\varepsilon
$$

which implies that $V+s \nsubseteq B$ for any $s \in S$. Hence, $B$ cannot contain arbitrarily large blocks, and the corollary is established.

4. Weak almost periodic functions on $\mathbf{N}$ and $\mathbf{Z}$. In this section we establish some necessary conditions for a function $f: \mathbf{N} \rightarrow \mathbf{R}$ to be weak almost periodic, and some sufficient ones. We then establish that there is a function in $W(\mathbf{N})$ which does not 
extend to a function in $W(\mathbf{Z})$. From this we conclude that the weak almost periodic compactification $\omega \mathbf{N}$ is not embedded in $\omega \mathbf{Z}$. Finally, some special properties of $\omega \mathbf{Z}$ are presented.

4.1 TheOREM. Let $f: \mathbf{N} \rightarrow \mathbf{R}$ be bounded, and assume that there exist $a \in \mathbf{R}$ and $\delta>0$ such that both

$$
A=\{x \in \mathbf{N} \mid f(x)<a-\delta\} \text { and } B=\{x \in \mathbf{N} \mid f(x)>a+\delta\}
$$

contain arbitrarily long blocks. Then $f \notin W(\mathbf{N})$.

Proof. This is a consequence of Corollary 3.7 and Comment 3.3.

The following theorem says that if $f$ is well behaved except on widely scattered blocks of bounded length, then $f$ is weak almost periodic.

4.2 THEOREM. Let $f: \mathbf{N} \rightarrow \mathbf{R}$ be bounded. Let $\left\langle a_{n}\right\rangle_{n=1}^{\infty}$ and $\left\langle b_{n}\right\rangle_{n=1}^{\infty}$ be sequences in $\mathbf{N}$ such that

(i) $a_{n}<b_{n}<a_{n+1}, n=1,2, \ldots$,

(ii) $\left\langle a_{n+1}-b_{n}\right\rangle_{n=1}^{\infty}$ is bounded, and

(iii) $\lim _{n \rightarrow \infty}\left(b_{n}-a_{n}\right)=\infty$.

And let

$$
D=\left\{x \in \mathbf{N} \mid a_{n} \leqslant x<b_{n}\right\} .
$$

If $\lim _{x \rightarrow \infty, x \in D} f(x)$ exists, then $f \in W(\mathbf{N})$.

Proof. Let $\left\langle t_{n}\right\rangle_{n=1}^{\infty}$ and $\left\langle s_{k}\right\rangle_{k=1}^{\infty}$ be sequences in $\mathbf{N}$ such that all the limits in

$$
\lim _{n \rightarrow \infty} \lim _{k \rightarrow \infty} f\left(t_{n}+s_{k}\right) \text { and } \lim _{k \rightarrow \infty} \lim _{n \rightarrow \infty} f\left(t_{n}+s_{k}\right)
$$

exist. Note that we may, by thinning, assume that $\left\langle t_{n}\right\rangle_{n=1}^{\infty}$ and $\left\langle s_{k}\right\rangle_{k=1}^{\infty}$ are increasing sequences. If either sequence is eventually constant, then the equality of the limits would follow immediately. So we assume that $\left\langle t_{n}\right\rangle_{n=1}^{\infty}$ and $\left\langle s_{k}\right\rangle_{k=1}^{\infty}$ are strictly increasing. Let

$$
c=\lim _{\substack{x \rightarrow \infty \\ x \in D}} f(x) .
$$

We show that both iterated limits are equal to $c$.

Suppose, instead, that $\lim _{n \rightarrow \infty} \lim _{k \rightarrow \infty} f\left(t_{n}+s_{k}\right) \neq c$. For each $n$, let $d_{n}=$ $\lim _{k \rightarrow \infty} f\left(t_{n}+s_{k}\right)$. If $d_{n} \neq c$, and we may assume that it does not, then for all but finitely many $k, t_{n}+s_{k} \notin D$. Pick $n$ and $m, n<m$, such that

$$
t_{m}-t_{n}>\max \left\{a_{r+1}-b_{r} \mid r \in \mathbf{N}\right\} \text {. }
$$

For each $k$ with $t_{n}+s_{k} \notin D$, pick $l(k)$ with

$$
b_{l(k)} \leqslant t_{n}+s_{k}<a_{l(k)+1} \text {. }
$$

Now pick $k$ so that $t_{n}+s_{k} \notin D, t_{m}+s_{k} \notin D$, and $b_{l(k)+1}-a_{l(k)+1}>t_{m}-t_{n}$. Then

$$
\begin{aligned}
t_{m}+s_{k} & =t_{n}+s_{k}+\left(t_{m}-t_{n}\right) \\
& >b_{l(k)}+a_{l(k)+1}-b_{l(k)}=a_{l(k)+1},
\end{aligned}
$$

while

$$
t_{m}+s_{k}=t_{n}+s_{k}+\left(t_{m}-t_{n}\right)<a_{l(k)+1}+b_{l(k)+1}-a_{l(k)+1}=b_{l(k)+1}
$$


which implies that $t_{m}+s_{k} \in D$, a contradiction. Therefore,

$$
\lim _{n \rightarrow \infty} \lim _{k \rightarrow \infty} f\left(t_{n}+s_{k}\right)=c \text {; }
$$

the proof that $\lim _{k \rightarrow \infty} \lim _{n \rightarrow \infty} f\left(t_{n}+s_{k}\right)=c$ is similar.

We now turn our attention to functions $f$ on $\mathbf{N}$ which take on only the values 0 and 1. From Theorem 4.1 we know that if $f^{-1}[\{0\}]$ and $f^{-1}[\{1\}]$ both include arbitrarily long blocks, then $f \notin W(\mathbf{N})$. From Theorem 4.2 we know that if, say, $f^{-1}[\{1\}]$ is contained in a sequence of blocks of bounded length and increasing separation, then $f \in W(\mathbf{N})$. The next two theorems concern the possibility that $f^{-1}[\{0\}]$ contains arbitrarily long blocks and $f^{-1}[\{1\}]$ is not contained in a sequence of blocks of bounded length.

4.3 Definition. Let $A \subseteq \mathbf{N}$, and let $z: \mathbf{N} \rightarrow \mathbf{N}$.

(a) $A$ is $z$-large if for each $n$ and $m$ in $N$, there exists $x \geqslant m$ such that whenever $t \in\{1,2, \ldots, n\}$, we have that the cardinality

$$
\operatorname{card}[A \cap\{x+1, x+2, \ldots, x+z(t)\}] \geqslant t .
$$

(b) $A$ has positive maximal density if there is some positive $\alpha$ in $\mathbf{R}$ such that for each $n$ and $m$ in $\mathbf{N}$, there exists $x \geqslant m$ with

$$
\operatorname{card}[A \cap\{x+1, x+2, \ldots, x+n\}] \geqslant n \alpha .
$$

The set $A$ has positive maximal density if and only if there is some function $z$ : $\mathbf{N} \rightarrow \mathbf{N}$ of the form $z(t)=k t$ such that $A$ is $z$-large [9, Theorem 2.2].

4.4 Theorem. Let $f: \mathbf{N} \rightarrow\{0,1\}$. If there is no function $z: \mathbf{N} \rightarrow \mathbf{N}$ such that $f^{-1}[\{1\}]$ is 3-large, then $f \in W(\mathbf{N})$.

Proof. Suppose $f \notin W(\mathbf{N})$. Pick sequences $\left\langle t_{n}\right\rangle_{n=1}^{\infty}$ and $\left\langle s_{k}\right\rangle_{k=1}^{\infty}$ so that

$$
\lim _{n \rightarrow \infty} \lim _{k \rightarrow \infty} f\left(t_{n}+s_{k}\right) \neq \lim _{k \rightarrow \infty} \lim _{n \rightarrow \infty} f\left(t_{n}+s_{k}\right) \text {. }
$$

Without loss of generality, assume

$$
\lim _{n \rightarrow \infty} \lim _{k \rightarrow \infty} f\left(t_{n}+s_{k}\right)=1 .
$$

We may further assume, by suitably thinning the sequences, that $f\left(t_{n}+s_{k}\right)=1$ whenever $n \leqslant k$. Then let $z(n)=t_{n}$.

4.5 TheOREM. Let $f: \mathbf{N} \rightarrow\{0,1\}$ such that $f^{-1}[\{0\}]$ contains arbitrarily large blocks. If $f^{-1}[\{1\}]$ has positive maximal density, then $f \notin W(\mathbf{N})$.

Proof. Let $A_{1}=f^{-1}[\{1\}]$. By [9, Theorem 3.7], if $A$ is a set with positive maximal density, then there exists $m \in \mathbf{N}$ such that for each $z \in \mathbf{N}$, there is some $t \in\{z+$ $1, z+2, \ldots, z+m\}$ such that $A \cap(A-t)$ has positive maximal density. Pick for each $n$ some $b_{n}$ such that

$$
\left\{b_{n}+1, b_{n}+2, \ldots, b_{n}+n\right\} \subseteq f^{-1}[\{0\}] .
$$

Pick $m_{1}$ as guaranteed above for $A_{1}$, let $z_{1}=b_{m_{1}+1}$ and pick $t_{1} \in\left\{z_{1}+1, z_{1}+\right.$ $\left.2, \ldots, z_{1}+m_{1}\right\}$ such that $A_{2}=A_{1} \cap\left(A_{1}-t_{1}\right)$ has positive maximal density. Inductively, given $A_{n}=A_{n-1} \cap\left(A_{n-1}-t_{n-1}\right)$ with positive maximal density, pick $m_{n}$ as guaranteed above, let $z_{n}=b_{m_{n}+n}$, and pick $t_{n} \in\left\{z_{n}+1, z_{n}+2, \ldots, z_{n}+m_{n}\right\}$ such 
that $A_{n+1}=A_{n} \cap\left(A_{n}-t_{n}\right)$ has positive maximal density. Note that, for each $n$, $\left\{t_{n}+1, t_{n}+2, \ldots, t_{n}+n\right\} \subseteq f^{-1}[\{0\}]$. For each $n$, choose $s_{n} \in A_{n} \cap\left(A_{n}-t_{n}\right)$. Note that

$$
s_{n} \in \bigcap_{k=1}^{n}\left(A_{1}-t_{k}\right)
$$

But then $\lim _{n \rightarrow \infty} \lim _{k \rightarrow \infty} f\left(t_{n}+s_{k}\right)=1$ and $\lim _{k \rightarrow \infty} \lim _{n \rightarrow \infty} f\left(t_{n}+s_{k}\right)=0$, and so $f \notin W(\mathbf{N})$.

In the next two results we are concerned with whether certain characteristic functions are weak almost periodic. These results are of interest because the weak almost periodic characteristic functions correspond to the clopen (i.e., open and closed) subsets of $\omega \mathbf{N}$. That is, if $\chi_{A} \in W(\mathbf{N})$, then $B=\operatorname{cl} \omega_{N}[A]$ is a clopen subset of $\omega \mathbf{N}$; and if $B$ is a given clopen subset of $\omega \mathbf{N}$ and $A=\omega_{\mathbf{N}}^{-1}[B]$, then $\chi_{A} \in W(\mathbf{N})$.

(Both assertions follow from Theorem 2.5.)

If $\left\langle x_{n}\right\rangle_{n=1}^{\infty}$ is a sequence in $\mathbf{N}$, then let

$$
P S\left(\left\langle x_{n}\right\rangle_{n=1}^{\infty}\right)=\left\{x_{n}+x_{m}: n \neq m\right\}
$$

and let

$$
F S\left(\left\langle x_{n}\right\rangle_{n=1}^{\infty}\right)=\left\{\sum_{n \in F} x_{n}: F \text { is finite and } F \neq \varnothing\right\} .
$$

Note that as an easy corollary to Theorem 4.6 we find that the characteristic function of the pairwise sums $P S\left(\left\langle p_{n}\right\rangle_{n=1}^{\infty}\right)$ of the sequence $\left\langle p_{n}\right\rangle_{n=1}^{\infty}$ of Fibonacci numbers is weak almost periodic. (That the characteristic function of the Fibonacci numbers $\left\{p_{n}: n=1,2, \ldots\right\}$ themselves is weak almost periodic is a consequence of Theorem 4.2.)

4.6 THEOREM. Let $\left\langle a_{n}\right\rangle_{n=1}^{\infty}$ be a sequence in $\mathbf{N}$ such that, for each $n$,

$$
a_{n+2} \geqslant a_{n}+a_{n+1} \text {. }
$$

Let $f$ be the characteristic function of $P S\left(\left\langle a_{n}\right\rangle_{n=1}^{\infty}\right)$. Then $f \in W(\mathbf{N})$.

Proof. Observe that $\left\langle a_{n}\right\rangle_{n=2}^{\infty}$ is increasing. Suppose we have sequences $\left\langle t_{n}\right\rangle_{n=1}^{\infty}$ and $\left\langle s_{k}\right\rangle_{k=1}^{\infty}$ in $\mathbf{N}$ such that

$$
\lim _{n \rightarrow \infty} \lim _{k \rightarrow \infty} f\left(t_{n}+s_{k}\right)=0 \text { and } \lim _{k \rightarrow \infty} \lim _{n \rightarrow \infty} f\left(t_{n}+s_{k}\right)=1 .
$$

We consider first the possibility that the set

$$
\left\{n \in \mathbf{N}:\left|t_{n}-a_{r}\right| \leqslant m \text { for some } r\right\}
$$

is infinite for some $m$. In such a case, by thinning suitably the sequences $\left\langle t_{n}\right\rangle_{n=1}^{\infty}$ and $\left\langle s_{n}\right\rangle_{n=1}^{\infty}$, we may assume that we have an $m \in \mathbf{Z}$ and, for each $n \in \mathbf{N}$, an $r(n)$ such that

(1) $t_{n}=a_{r(n)}+m$ for each $n$,

(2) $\lim _{n \rightarrow \infty} f\left(t_{n}+s_{k}\right)=1$ for each $k$,

(3) $s_{k}>|m|$ for each $k$, and

(4) the sequences $\left\langle t_{n}\right\rangle_{n=1}^{\infty}$ and $\left\langle s_{n}\right\rangle_{n=1}^{\infty}$ are increasing sequences.

Now let $k$ be given, and pick $n$ such that

$$
a_{r(n)-1} \geqslant m+s_{k} \text { and } f\left(t_{n}+s_{k}\right)=1 \text {. }
$$


Then $t_{n}+s_{k}=a_{i}+a_{j}$ for some $j<i$. Thus $t_{n}+s_{k} \leqslant a_{i}+a_{i-1} \leqslant a_{i+1}$, so that $t_{n} \leqslant a_{i+1}-s_{k}<a_{i+1}+m$. Thus $i \geqslant r(n)$. Also,

$$
a_{i}<t_{n}+s_{k}=a_{r(n)}+m+s_{k} \leqslant a_{r(n)}+a_{r(n)-1},
$$

so that $i \leqslant r(n)$; and, hence, $i=r(n)$. That yields,

$$
a_{r(n)}+m+s_{k}=a_{r(n)}+a_{j} \text {. }
$$

We, therefore, have for each $k$, some $j(k)$ such that $s_{k}=a_{j(k)}-m$. But then

$$
\lim _{n \rightarrow \infty} \lim _{k \rightarrow \infty} f\left(t_{n}+s_{k}\right)=1
$$

a contradiction.

Thus, we must have that for each $m$, the set

$$
A_{m}=\left\{n \in \mathbf{N}:\left|t_{n}-a_{r}\right| \leqslant m \text { for some } r\right\}
$$

is finite. We may assume that $s_{1}<s_{2}, \lim _{n \rightarrow \infty} f\left(t_{n}+s_{1}\right)=1$ and $\lim _{n \rightarrow \infty} f\left(t_{n}+s_{2}\right)$ $=1$; in fact, we may assume that for all $n$,

$$
f\left(t_{n}+s_{1}\right)=f\left(t_{n}+s_{2}\right)=1 \text {. }
$$

Pick for each $n, \alpha(n)>\beta(n)$ and $\gamma(n)>\delta(n)$ such that

$$
t_{n}+s_{1}=a_{\alpha(n)}+a_{\beta(n)} \text { and } t_{n}+s_{2}=a_{\gamma(n)}+a_{\delta(n)} .
$$

Note that $\alpha(n) \leqslant \gamma(n)$ since, otherwise,

$$
\begin{aligned}
s_{1} & =a_{\alpha(n)}+a_{\beta(n)}-a_{\gamma(n)}-a_{\delta(n)}+s_{2} \\
& \geqslant a_{\gamma(n)+1}+a_{\beta(n)}-a_{\gamma(n)}-a_{\delta(n)}+s_{2} \\
& \geqslant a_{\gamma(n)-1}+a_{\beta(n)}-a_{\delta(n)}+s_{2} \\
& >a_{\gamma(n)-1}-a_{\delta(n)}+s_{2} \geqslant s_{2} .
\end{aligned}
$$

Now assume $\alpha(n)<\gamma(n)$. Then

$$
\begin{aligned}
s_{2}-s_{1} & =a_{\gamma(n)}+a_{\delta(n)}-a_{\alpha(n)}-a_{\beta(n)} \\
& \geqslant a_{\gamma(n)}+a_{\delta(n)}-a_{\alpha(n)}-a_{\alpha(n)-1} \\
& \geqslant a_{\gamma(n)}+a_{\delta(n)}-a_{\alpha(n)+1} \geqslant a_{\delta(n)} .
\end{aligned}
$$

Thus, if infinitely often $\alpha(n)<\gamma(n)$, the sequence $\langle\delta(n)\rangle_{n=1}^{\infty}$ repeats infinitely often. But

$$
\left|t_{n}-a_{\gamma(n)}\right|=\left|a_{\delta(n)}-s_{2}\right| \leqslant a_{\delta(n)}+s_{2},
$$

so such repetition contradicts the assumption that $A_{m}$ is finite, $m=a_{\delta(n)}+s_{2}$. Thus for all but finitely many $n$, we have $\alpha(n)=\gamma(n)$, and hence

$$
t_{n}+s_{1}=a_{\alpha(n)}+a_{\beta(n)} \text { and } t_{n}+s_{2}=a_{\alpha(n)}+a_{\delta(n)} .
$$

Thus, for these $n$,

$$
s_{2}-s_{1}=a_{\delta(n)}-a_{\beta(n)} \geqslant a_{\delta(n)}-a_{\delta(n)-1} .
$$

But since $\lim _{n \rightarrow \infty}\left(a_{n+1}-a_{n}\right)=\infty$, this again forces the sequence $\langle\delta(n)\rangle_{n=1}^{\infty}$ to repeat infinitely often, which we have already seen to be impossible.

By way of contrast to Theorem 4.6, if we again require a relatively large separation of the terms of a sequence but now take finite sums instead of pairwise sums, we 
obtain the opposite conclusion. Finite sums in $\mathbf{N}$ are intimately related to idempotents in $(\beta \mathbf{N},+)$. Specifically, a subset $A$ of $\mathbf{N}$ is a member of some idempotent ultrafilter if and only if $F S\left(\left\langle a_{n}\right\rangle_{n=1}^{\infty}\right) \subseteq A$ for some sequence $\left\langle a_{n}\right\rangle_{n=1}^{\infty}$ in $\mathbf{N}$ [11, Corollary 10.16]. (That result is due to F. Galvin.)

4.7 Theorem. Let $\left\langle a_{n}\right\rangle_{n=1}^{\infty}$ be an increasing sequence in $\mathbf{N}$, and let $f$ be the characteristic function of $F S\left(\left\langle a_{n}\right\rangle_{n=1}^{\infty}\right)$. If for each $m$, there is some $n$ such that

$$
a_{n+1} \geqslant m+\sum_{j=1}^{n} a_{j}
$$

then $f \notin W(\mathbf{N})$.

Proof. Inductively choose sequences $\langle\delta(n)\rangle_{n=1}^{\infty}$ and $\langle\gamma(n)\rangle_{n=1}^{\infty}$ in $\mathbf{N}$ such that for each $n$

(1) $\delta(n+1)>\delta(n)$ and $\delta(n) \notin\{\gamma(j): 1 \leqslant j<n\}$,

(2) $\gamma(n+1)>\gamma(n)+1, \gamma(n+1)>\delta(n)$, and

(3) $a_{\gamma(n)} \geqslant a_{\delta(n)}+\sum_{j=1}^{\gamma(n)-1} a_{j}$.

Let $L=\{\gamma(n): n \in \mathbf{N}\}$, and, for each $n$, let $F(n)=\{1,2,3, \ldots, n\} \backslash L$. For $n \in \mathbf{N}$, let $t_{n}=a_{\gamma(n)}-a_{\delta(n)}$, and let $s_{n}=\Sigma_{j \in F(n)} a_{j}$.

Now, given $n$ and $k>\delta(n)$, let

$$
G(n, k)=(F(k) \backslash\{\delta(n)\}) \cup\{\gamma(n)\} .
$$

Then, since $\delta(n) \notin L$ and $\gamma(n) \in L$, we have

$$
t_{n}+s_{k}=\sum_{j \in G(n, k)} a_{j},
$$

so $f\left(t_{n}+s_{k}\right)=1$. Thus, for each $n$,

$$
\lim _{k \rightarrow \infty} f\left(t_{n}+s_{k}\right)=1 .
$$

Now given $k$ and $n$ such that $a_{\delta(n)}>s_{k}$, we claim that $f\left(t_{n}+s_{k}\right)=0$. Suppose, instead, that $f\left(t_{n}+s_{k}\right)=1$; which is to say, suppose that $t_{n}+s_{k} \in F S\left(\left\langle a_{n}\right\rangle_{n=1}^{\infty}\right)$, or that

$$
t_{n}+s_{k}=\sum_{j \in G} a_{j}
$$

for some finite $G \subseteq \mathbf{N}$. Then

$$
\sum_{j \in G} a_{j}=a_{\gamma(n)}-a_{\delta(n)}+s_{k}<a_{\gamma(n)} ;
$$

so $\max G<\gamma(n)$. But then

$$
s_{k}=\sum_{j \in G} a_{j}-a_{\gamma(n)}+a_{\delta(n)} \leqslant \sum_{j=1}^{\gamma(n)-1} a_{j}-a_{\gamma(n)}+a_{\delta(n)} \leqslant 0 .
$$

But $F(k) \neq \varnothing ;$ so $s_{k}>0$, a contradiction. 
We suspect that the condition on $\left\langle a_{n}\right\rangle_{n=1}^{\infty}$, imposed by Theorem 4.7, may be both necessary and sufficient for the characteristic function of $F S\left(\left\langle a_{n}\right\rangle_{n=1}^{\infty}\right)$ to fail to be weak almost periodic. At any rate it is true that if there is some $m \in \mathbf{N}$ such that for each $n$,

$$
a_{n+1}=m+\sum_{j=1}^{n} a_{j},
$$

then the characteristic function $f$ of $F S\left(\left\langle a_{n}\right\rangle_{n=1}^{\infty}\right)$ is weak almost periodic. (For then, if $n \geqslant 2, a_{n}=2^{n-2} a_{2}$, and hence $f(x)=1$ if and only if $x$ is congruent to either 0 or $a_{1} \bmod a_{2}$.)

The next result is the major result of this section. A bounded continuous complex-valued function $f$ on a semitopological semigroup $S$ is almost periodic if the norm closure $\operatorname{cl}\left\{f_{s}: s \in S\right\}$ is compact in $C(S)$. The proof of [4, Corollary 2.30, p. 35] applied to $\mathbf{Z}$ and $\mathbf{N}$ instead of $\mathbf{R}$ and $\mathbf{R}^{+}$establishes that

$$
A(\mathbf{N})=\left.C_{0}(\mathbf{N}) \oplus A(\mathbf{Z})\right|_{\mathbf{N}} .
$$

Since $C_{0}(\mathbf{Z}) \subseteq W(\mathbf{Z})$ [4, Corollary 3.7, p. 41], and every function vanishing at infinity on $\mathbf{N}$ can be extended as a $C_{0}$ function to $\mathbf{Z}$, we see that every almost periodic function on $\mathbf{N}$ extends as a weak almost periodic function on $\mathbf{Z}$, though not as an almost periodic function because no nontrivial almost periodic function on a noncompact, locally compact group can vanish at infinity [4, Corollary 3.8, p. 41]. A tempting conjecture would be that each $f \in W(\mathbf{N})$ extends as a weak almost periodic function on $\mathbf{Z}$.

4.8 THEOREM. $\left.W(\mathbf{Z})\right|_{\mathbf{N}} \subsetneq W(\mathbf{N})$.

Proof. Let $f(x)=1$ if there exist $m, r, n \in \mathbf{N}$ with $m \leqslant n$ such that

$$
x=2^{2^{n}(2 r+1)}-2^{m},
$$

and $f(x)=0$ otherwise. We show first that $f \in W(\mathbf{N})$. Suppose, instead, we have sequences $\left\langle t_{n}\right\rangle_{n=1}^{\infty}$ and $\left\langle s_{n}\right\rangle_{n=1}^{\infty}$ in $\mathbf{N}$ such that $\lim _{n \rightarrow \infty} \lim _{k \rightarrow \infty} f\left(t_{n}+s_{k}\right)=0$ and $\lim _{k \rightarrow \infty} \lim _{n \rightarrow \infty} f\left(t_{n}+s_{k}\right)=1$. We may, and do, assume that each sequence is increasing and that, given $n$ and $k \leqslant n, f\left(t_{n}+s_{k}\right)=1$. For each $l \geqslant 2$, choose $n(l, 1), n(l, 2), m(l, 1), m(l, 2), r(l, 1)$ and $r(l, 2)$ so that with

$$
a(l)=2^{n(l, 1)-1}(2 r(l, 1)+1) \text { and } b(l)=2^{n(l, 2)-1}(2 r(l, 2)+1),
$$

we have

$$
t_{l}+s_{1}=2^{2 a(l)}-2^{m(l, 1)} \text { and } t_{l}+s_{2}=2^{2 b(l)}-2^{m(l, 2)} .
$$

(Here $m(l, 1) \leqslant n(l, 1)$ and $m(l, 2) \leqslant n(l, 2)$.) Note that $m(l, 1)<a(l)$ and $m(l, 2)<$ $b(l)$. Note further that as $l$ increases, $a(l)$ assumes any one value only finitely often. (Otherwise, since $m(l, 1)<a(l)$ one would have $t_{l}+s_{1}$ repeating.) Thus for infinitely many $l$ we have $2^{2 a(l)}>s_{2}-s_{1}$. For such $l$, we claim that $a(l)=b(l)$. Since $s_{1}<s_{2}$, we get

$$
2^{2 a(l)}+2^{m(l, 2)}<2^{2 b(l)}+2^{m(l, 1)}
$$


so that $a(l) \leqslant b(l)$. Suppose now that $b(l) \geqslant a(l)+1$. Then

$$
\begin{aligned}
s_{2}-s_{1} & =2^{2 b(l)}-2^{m(l, 2)}-2^{2 a(l)}+2^{m(l, 1)} \\
& >2^{2 b(l)}-2^{b(l)}-2^{2 a(l)}>2^{2 b(l)-1}-2^{2 a(l)} \\
& \geqslant 2^{2 a(l)+1}-2^{2 a(l)}=2^{2 a(l)},
\end{aligned}
$$

a contradiction.

We thus have, for $2^{2 a(l)}>s_{2}-s_{1}$, that $t_{l}+s_{1}=2^{2 a(l)}-2^{m(l, 1)}$ and $t_{l}+s_{2}=$ $2^{2 a(l)}-2^{m(l, 2)}$. Now we note, given such $l$, that $s_{2}-s_{1}=2^{m(l, 1)}-2^{m(l, 2)}$ so that $m(l, 1)$ and $m(l, 2)$ are independent of $l$. (A given member of $\mathbf{N}$ can be expressed in at most one way as a difference of powers of 2.) Thus we have $u$ and $v$ in $\mathbf{N}$ such that $t_{l}+s_{1}=2^{2 a(l)}-2^{u}$ and $t_{l}+s_{2}=2^{2 a(l)}-2^{v}$ whenever $2^{2 a(l)}>s_{2}-s_{1}$. Now pick $k$ such that $s_{k}-s_{1}>2^{u}$. Again, as above we have some $x \in \mathbf{N}$ such that $t_{l}+s_{k}=$ $2^{2 a(l)}-2^{x}$ whenever $2^{2 a(l)}>s_{k}-s_{1}$. But then, $2^{2 a(l)}>t_{l}+s_{k}>t_{l}+s_{1}+2^{u}=$ $2^{2 a(l)}-2^{u}+2^{u}$, a contradiction.

We now show that $f$ cannot be extended to a member, say $g$, of $W(\mathbf{Z})$. Suppose we have such $g$ and consider $B=\left\{-2^{m}: m \in \mathbf{N}\right\}$. Let $B_{1}=B$. Inductively let $C_{t, 1}=$ $\left\{s \in B_{t}:|g(s+t)|<\frac{1}{2}\right\}$ and let $C_{t, 2}=B_{t} \backslash C_{t, 1}$. Pick $i \in\{1,2\}$ such that $C_{t, i}$ is infinite and let $B_{t+1}=C_{t, i}$. Now let $s_{1} \in B_{1}$ and for $k \in \mathbf{N}$, pick $s_{k+1}<s_{k}$. Then, given $k, n$ and $t$ in $\mathbf{N}$ with $t \leqslant k \leqslant n$, we have $\left|g\left(s_{k}+t\right)-1\right|<\frac{1}{2}$ if and only if

$$
\left|g\left(s_{n}+t\right)-1\right|<\frac{1}{2} \text {. }
$$

Let $A=\left\{t \in \mathbf{N}:\left|g\left(s_{t}+t\right)-1\right|<\frac{1}{2}\right\}$. We consider first the possibility that for each $n \in \mathbf{N}$ there is some $r \in \mathbf{N}$ with $2^{2^{n}(2 r-1)} \notin A$. Then for each $n$ pick such $r(n)$ and let $t_{n}=2^{2^{n}(2 r-1)}$. Since each $t_{n} \notin A$, we have (after thinning the sequences so that the limit exists) that $\lim _{n \rightarrow \infty} \lim _{k \rightarrow \infty} g\left(t_{n}+s_{k}\right) \neq 1$. On the other hand, given $s_{k}=$ $-2^{m}$, we have for $n \geqslant m$ that $g\left(t_{n}+s_{k}\right)=f\left(t_{n}+s_{k}\right)=1$ so

$$
\lim _{k \rightarrow \infty} \lim _{n \rightarrow \infty} g\left(t_{n}+s_{k}\right)=1 \text {, }
$$

a contradiction.

We therefore pick $n \in \mathbf{N}$ such that $\left\{2^{2^{n}(2 r+1)}: r \in \mathbf{N}\right\} \subseteq A$. For each $r \in \mathbf{N}$, let $t_{r}=2^{2^{n}(2 r+1)}$. Then each $t_{r} \in A$ so (again after thinning) $\lim _{r \rightarrow \infty} \lim _{k \rightarrow \infty} g\left(t_{r}+s_{k}\right)$ $=a$, where $|a-1| \leqslant \frac{1}{2}$. Now let $k$ be given with $s_{k}=-2^{m}$ where $m>n$. Then, if $2^{n}(2 r+1)>m$, we have that $t_{r}+s_{k}=2^{2^{n}(2 r+1)}-2^{m}$ and (again since a member of $\mathbf{N}$ can be expressed in at most one way as a difference of powers of 2) that $f\left(t_{r}+s_{k}\right)=0$. Thus $\lim _{k \rightarrow \infty} \lim _{r \rightarrow \infty} g\left(t_{r}+s_{k}\right)=0$, a contradiction.

Every compact right topological semigroup $S$ contains a minimal (two-sided) ideal whose algebraic structure is well understood [3, Theorem II. 2.2, p. 50]. If $S$ is an abelian semitopological semigroup, then $\omega S$ is abelian and, therefore, has a compact topological group $K(\omega S)$ for a minimal ideal [2, Lemma II. 3.1(iv), p. 65 and II. 3.3, p. 66]. In particular, $K(\omega \mathbf{N})$ and $K(\omega \mathbf{Z})$ are compact topological groups; in fact, $K(\omega \mathbf{N})$ and $K(\omega \mathbf{Z})$ are both isomorphic to the largest compact monothetic group [8, Theorem 25.12, p. 407]. The following theorems shed some light on how this group is embedded in $\omega \mathbf{N}$ and $\omega \mathbf{Z}$. A strong almost periodic function is the uniform limit of linear combinations of characters (homomorphisms into the circle group $\mathbf{T}$ ). 
4.9 THEOREM. A function is strong almost periodic on $(\mathbf{Z},+)$ if and only if it is almost periodic. A function is strong almost periodic on $(\mathbf{N},+)$ if and only if it is the restriction to $\mathbf{N}$ of an almost periodic function on $\mathbf{Z}$. Let $S$ be $(\mathbf{Z},+)$ or $(\mathbf{N},+)$. Let e be the identity of the group $K(\omega S)$, and let $U=\{f \in W(S)$ : $f$ is strong almost periodic $\}$. Then the continuous function

$$
\rho_{e}(t)=t+e: \omega S \rightarrow K(\omega S)
$$

is a homomorphism and a retract. Furthermore, for $f \in W(S)$,

$$
f^{\omega}(t+e)=f^{\omega}(t), \quad \forall t \in \omega S,
$$

if and only if $f \in U$.

Proof. The first assertion results from $(\mathrm{Z},+)$ being a group [3, III. 14.5(v), p. 127]. The second assertion is a direct result of [4, Theorem 2.29, p. 33]. The last assertion follows directly from [1, Proposition 5, p. 285].

The homomorphism $\rho_{e}$ of Theorem 4.9 is called the Clifford-Miller endomorphism [12, Definition A.2.8, p. 18]. Any homomorphism $\eta$ on a semigroup $S$ induces a congruence relation on $S$. (A congruence relation is an equivalence relation $\sim$ which satisfies

$$
x \sim y \text { implies } s+x \sim s+y \text { and } x+s \sim y+s
$$

for every $s \in S$.) Thus we have a congruence on $\omega S$, for any commutative semigroup $S$, given by $s \sim t$ if and only if $s+e=t+e$. By Theorem 4.9, then, if $S$ is $(\mathbf{N},+)$ or $(\mathbf{Z},+)$ we have that $s \sim t$ if and only if $f^{\omega}(s)=f^{\omega}(t)$ for every $f \in U$. But this is equivalent to saying $s \sim t$ if and only if $f^{\omega}(s)=f^{\omega}(t)$ for every character $f$ on $S$. A character on $S$ is uniquely determined by its value at 1 : if $f(1)=e^{i \theta}, 0 \leqslant \theta<2 \pi$, then $f(n)=e^{i n \theta}$. Now $\omega_{S}(0)$ and $e$ are in the same equivalence class, and from the above we get that a net $n_{\alpha}$ is such that

$$
e \sim \lim \omega_{S}\left(n_{\alpha}\right)
$$

if and only if

$$
\lim e^{i n_{\alpha} \theta}=1 \text { for every } \theta \in[0,2 \pi) .
$$

5. Compactifications of $S$ as spaces of filters. Recall from Theorem 2.4 that if $(S,+)$ is a discrete semigroup, $(\beta S,+)$ is the maximal left topological semigroup extension of $S$. Thus given any compact left topological semigroup $(T,+)$ and a homomorphism $f: S \rightarrow T$ such that $\rho_{f(s)}$ is continuous for each $s \in S$ and $f[S]$ is dense in $T$, we have $f^{\beta}$ is a homomorphism of $(\beta S,+)$ onto $(T,+)$. (There is some continuous homomorphism from $\beta S$ onto $T$ which extends $f$, but $f^{\beta}$ was the only continuous extension of $f$.) Consequently, $T$ is a topological quotient of $\beta S$. (Let $R(f)=\left\{(p, q): p, q \in \beta S\right.$ and $\left.f^{\beta}(p)=f^{\beta}(q)\right\}$. Then $T \approx \beta S / R(f)$ via the homomorphism $g([p])=f(p)$. Further, defining + on $\beta S / R(f)$ by $[p]+[q]=[p+q]$ we have $g$ is an isomorphism of $(\beta S / R(f),+)$ onto $(T,+)$.)

Given an equivalence relation $R$ on $\beta S$ such that $\beta S / R$ is Hausdorff, we have the $R$-equivalence classes are closed subsets of $\beta S$. But the closed subsets of $\beta S$ correspond exactly to the filters on $S$. 


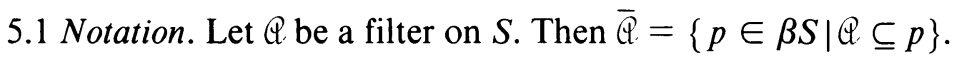

The correspondence between the closed subset of $\beta S$ and the filters of $S$ is as follows. Given any subset $A$ of $\beta S, \cap A$ is a filter on $S$ (since it is the intersection of a set of ultra filters) and $\overline{\cap A}=\mathrm{cl} A$. We thus have that any left topological compactification of $S$ corresponds to a set of filters on $S$. In this section we characterize those sets of filters which yield left topological compactifications of $S$ and describe the topology and the operation in terms of the filters. The results through Corollary 5.12 do not refer to any algebraic structure. We assume merely that $S$ is a set with the discrete topology.

Our first objective is to determine when a set $\Re$ of filters on $S$ corresponds to a quotient of $\beta S$ by closed equivalence classes. Conditions (a) and (b) of the following theorem provide a description, internal to $R$, of precisely when this happens. (In practice condition $\left(a^{\prime}\right)$ is easier to work with than condition (a).)

5.2 THEOREM. Let $\Re$ be a set of filters on S. Statements (a) and ( $\left.\mathrm{a}^{\prime}\right)$ are equivalent, statements $(\mathrm{b})$ and $\left(\mathrm{b}^{\prime}\right)$ are equivalent, and statement $(\mathrm{c})$ is equivalent to the conjunction of statements (a) and (b).

(a) Given any choice function $f$ for $\mathcal{R}$ there is a finite subfamily $\mathcal{F}$ of $\mathcal{R}$ such that $S=\cup_{Q \in \mathscr{F}} f(\mathbb{Q})$.

(a') For each $p \in \beta S$ there is some $\mathbb{Q} \in \mathcal{R}$ such that $\mathbb{Q} \subseteq p$.

(b) Given distinct $\mathbb{Q}$ and $\mathscr{B}$ in $\mathcal{R}$, there exists $B \in \mathscr{B}$ such that $S \backslash B \in \mathbb{Q}$.

(b') For each $p \in \beta S$ there is at most one $\mathbb{Q} \in \mathcal{R}$ such that $\mathbb{Q} \subseteq p$.

(c) There is an equivalence relation $R$ on $\beta S$ such that each equivalence class is closed in $\beta S$ and $R=\left\{\cap[p]_{R}: p \in \beta S\right\}$.

Proof. To see that $\left(\mathrm{a}^{\prime}\right)$ implies (a) let $f$ be a choice function for $R$. (That is, $f$ : $\Re \rightarrow \cup \Re$ and for each $\mathscr{Q} \in R, f(\mathbb{Q}) \in \mathbb{Q}$.) Suppose that the conclusion of (a) fails. Then $\{S \backslash f(\mathbb{Q}): \mathbb{Q} \in \mathcal{R}\}$ has the finite intersection property so pick $p \in \beta S$ such that $\{S \backslash f(\mathbb{Q}): \mathbb{Q} \in \mathscr{R}\} \subseteq p$. Pick $\mathscr{Q} \in \mathcal{R}$ such that $\mathscr{Q} \subseteq p$. Then $f(\mathbb{Q}) \in p$ and $S \backslash f(\mathbb{Q}) \in p$, a contradiction.

To see that (a) implies $\left(a^{\prime}\right)$ let $p \in \beta S$ and suppose that the conclusion of $\left(a^{\prime}\right)$ fails. For each $Q \in \mathcal{Q}$, pick $f(\mathbb{Q}) \in \mathbb{Q} \backslash p$. Pick a finite subfamily $\mathscr{F}$ of $\Re$ such that $S=\cup_{\mathscr{Q} \in \mathscr{F}} f(\mathbb{Q})$. But then for some $\mathbb{Q} \in \mathcal{F}, f(\mathbb{Q}) \in p$, a contradiction.

To see that $\left(b^{\prime}\right)$ implies (b) let $\mathscr{Q}$ and $\mathscr{G}$ be distinct members of $R$ and suppose that for each $B \in \mathscr{B}, S \backslash B \notin \mathbb{Q}$. Then for each $B \in \mathscr{B}, \mathbb{Q} \cup\{B\}$ has the finite intersection property. (If $\mathscr{F}$ is a finite subfamily of $\mathcal{Q}$ and $B \cap(\cap \mathscr{F})=\varnothing$, then $\cap \mathscr{F} \subseteq S \backslash B$.) Thus, since $\mathscr{B}$ is closed under finite intersection, $Q \cup \mathscr{B}$ has the finite intersection property. But then one can pick $p \in \beta S$ such that $Q \cup \Re \subseteq p$ contradicting $\left(\mathrm{b}^{\prime}\right)$.

To see that (b) implies $\left(\mathrm{b}^{\prime}\right)$, let $p \in \beta S$ and suppose we have distinct $\mathcal{Q}$ and $\mathscr{B}$ in $\Re$ such that $Q \cup \mathscr{B} \subseteq p$. Pick $B \in \mathbb{B}$ such that $S \backslash B \in \mathbb{Q}$. Then $B \in p$ and $S \backslash B \in p$, a contradiction.

That (c) implies $\left(\mathrm{a}^{\prime}\right)$ is trivial since $\cap[p] \subseteq p$. To see that (c) implies $\left(\mathrm{b}^{\prime}\right)$ suppose we have $q \in \beta S$ such that $[q] \neq[p]$ and $\cap[q] \subseteq p$. Since $[q]$ is closed in $\beta S$ and $p \notin[q]$, pick $A \in p$ such that $\bar{A} \cap[q]=\varnothing$. Then $S \backslash A \in \cap[q]$ so $S \backslash A \in p$, a contradiction. 
Now assume that $\left(\mathrm{a}^{\prime}\right)$ and $\left(\mathrm{b}^{\prime}\right)$ hold. Define $R$ on $\beta S$ by $p R q$ if and only if there exists $Q \in R$ such that $Q \subseteq p \cap q$. Trivially $R$ is symmetric. By $\left(\mathrm{a}^{\prime}\right), R$ is reflexive on $\beta S$. To see that $R$ is transitive, let $p, q$ and $r$ be in $\beta S$ and assume that $p R q$ and $q R r$. Pick $\mathbb{Q}$ and $\mathscr{B}$ in $\mathscr{R}$ such that $\mathscr{Q} \subseteq p \cap q$ and $\mathscr{B} \subseteq q \cap r$. Since $\mathscr{Q} \cup \mathscr{B} \subseteq q$ we have by $\left(b^{\prime}\right)$ that $\mathbb{P}=\mathscr{B}$.

To see that $\mathscr{R}=\left\{\cap[p]_{R}: p \in \beta S\right\}$, it suffices to show that if $\mathscr{Q} \in \mathcal{R}$ and $p \in \beta S$ such that $\mathbb{Q} \subseteq p$, then $\mathcal{Q}=\cap[p]$. (For given any $\mathbb{Q} \in \mathcal{R}$ there is some $p \in \beta S$ such that $Q \subseteq p$ and given $p \in \beta S$ we have by $\left(\mathrm{a}^{\prime}\right)$ some $\mathcal{Q} \in \mathcal{R}$ such that $\mathcal{Q} \subseteq p$.) Let $\mathbb{Q} \in \mathscr{R}$ and $p \in \beta S$ with $\mathscr{Q} \subseteq p$ be given. Given $q \in[p]$ there is some $\mathscr{B} \in \mathscr{R}$ such that $\mathscr{B} \subseteq p \cap q$. But then, by $\left(\mathrm{b}^{\prime}\right), \mathscr{B}=\mathscr{Q}$ so $\mathscr{Q} \subseteq q$. Thus $\mathscr{Q} \subseteq \cap[p]$. To see that $\cap[p] \subseteq \mathbb{Q}$, let $A \in \cap[p]$ and suppose that $A \notin \mathbb{Q}$. Then $\mathcal{Q} \cup\{S \backslash A\}$ has the finite intersection property, so pick $q \in \beta S$ such that $\mathbb{U} \cup\{S \backslash A\} \subseteq q$. But then $\mathbb{Q} \subseteq p \cap q$ so $q \in[p]$ and hence $A \in q$, a contradiction.

Finally to show that each $R$-equivalence class is closed in $\beta S$ let $p \in \beta S$ and pick $Q \in R$ such that $Q \subseteq p$. Then $\overline{\mathscr{Q}}=[p]$ and, as we have observed, $\overline{\mathscr{Q}}$ is closed in $\beta S$.

5.3 Notation. Let $A \subseteq S$ and let $\Re$ be a set of filters on $S$. Then

$$
A^{*}=A^{*}(\Re)=\{\mathbb{R} \in \Re \mid A \in \mathbb{Q}\} \text {. }
$$

Give $\mathcal{R}$ and given $A, B \subseteq S$ we have $A^{*} \cap B^{*}=(A \cap B)^{*}$ and hence $\left\{A^{*}\right.$ : $A \subseteq S\}$ is a basis for some topology on $\Re$. Lemma 5.5 justifies the terminology of the following definition.

5.4 Definition. Let $R$ be a set of filters on $S$. The quotient topology on $R$ is the topology generated by $\left\{A^{*}: A \subseteq S\right\}$.

5.5 LEMMA. Let $R$ be an equivalence relation on $\beta S$ such that $\beta S / R$ is Hausdorff. Let $R=\left\{\cap[p]_{R}: p \in \beta S\right\}$. Then, with the quotient topology on $\Re, \Re \approx \beta S / R$.

Proof. Define $f: \beta S / R \rightarrow \Re$ by $f([p])=\bigcap[p]$. Then $f$ is trivially onto $R$. To see that $f$ is one-to-one, let $p, q \in \beta S$ such that $[p] \neq[q]$. Then $q \notin[p]$ and, since $\beta S / R$ is Hausdorff, $[p]$ is closed in $\beta S$. Pick $A \in q$ such that $\bar{A} \cap[p]=\varnothing$. Then $S \backslash A \in(\cap[p]) \backslash(\cap[q])$ so $f([p]) \neq f([q])$.

To see that $f$ is continuous, let $A \subseteq S$, so that $A^{*}$ is a basic open set in $R$. Let $U=\{[q]: q \in \beta S$ and $A \in \cap[q]\}$. Since " $A \in \cap[q]$ " and " $\cap[q] \in A^{*}$ " are synonymous, we have directly that $U=f^{-1}\left[A^{*}\right]$. We thus need to show that $U$ is open in $\beta S / R$. Let $g$ denote the projection from $\beta S$ to $\beta S / R$. Now given $q \in \beta S$, $A \in \cap[q]$ if and only if whenever $p R q, A \in p$, or equivalently $S \backslash A \notin p$. That is, $U=(\beta S / R) \backslash g[\overline{S \backslash A}]$. Now $\overline{S \backslash A}$ is closed in $\beta S$, hence compact. Thus $g[\overline{S \backslash A}]$ is the continuous image of a compact set, hence compact. Since $\beta S / R$ in Hausdorff, $g[\overline{S \backslash A}]$ is closed. That is, $U$ is open as required.

To see that $f$ is an open map, let $V$ be open in $\beta S / R$ and let $[p] \in V$. We produce $B \subseteq S$ such that $\cap[p] \in B^{*}$ and $B^{*} \subseteq f[V]$. For each $q \in[p], q \in g^{-1}[V]$, so pick $B_{q} \in q$ such that $\bar{B}_{q} \subseteq g^{-1}[V]$. Then $\left\{\bar{B}_{q}: q \in[p]\right\}$ is an open cover of the closed subset $[p]$ of $\beta S$. Pick finite $\mathscr{F} \subseteq[p]$ so that $[p] \subseteq \cup_{q \in \mathscr{F}} \bar{B}_{q}$ and let $B=\cup_{q \in \mathscr{F}} B_{q}$. Then $[p] \subseteq \cup_{q \in \mathscr{F}} \overline{B_{q}}=\bar{B} \subseteq g^{-1}[V]$. Since $[p] \subseteq \bar{B}, B \in \cap[p]$ and hence $\cap[p] \in$ $B^{*}$. To see that $B^{*} \subseteq f[V]$, let $\cap[r] \in B^{*}$. Then $B \in r$ so $r \in g^{-1}[V]$. That is, $[r] \in V$ so $\cap[r] \in f[V]$. 
5.6 THEOREM. Let $\Re$ be a set of filters on $S$ with the quotient topology. There is an equivalence relation $R$ on $\beta S$ such that $\beta S / R$ is Hausdorff, $R=\left\{\cap[p]_{R}: p \in \beta S\right\}$, and $R \approx \beta S / R$ if and only if both of the following statements hold.

(a) Given any choice function $f$ for $R$, there is a finite subfamily $\mathcal{F}$ of $R$ such that $S=\cup_{\mathbb{Q} \in \mathcal{F}} f(\mathbb{Q})$.

(b) Given distinct $\mathcal{Q}$ and $\mathscr{B}$ in $\mathscr{R}$, there exist $A \in \mathbb{Q}$ and $B \in \mathscr{B}$ such that, whenever $C \in \Re$, either $S \backslash A \in \mathcal{C}$ or $S \backslash B \in \mathcal{C}$.

Proof. (Necessity) Statement (a) holds by Theorem 5.2. To establish statement (b) let $\mathcal{Q}$ and $\Re$ be distinct members of $R$. Note that since $R \approx \beta S / R, \mathcal{R}$ is compact and Hausdorff. Pick disjoint basic neighborhoods $F^{*}$ and $G^{*}$ of $\mathcal{Q}$ and $\mathscr{B}$, respectively. For each $C \in \Re \backslash F^{*}$ pick, by Theorem 5.2, $E(\mathcal{C}) \in \mathcal{C}$ such that $S \backslash E(\mathcal{C}) \in \mathbb{Q}$. Then $R \backslash F^{*} \subseteq \cup\left\{E(\mathcal{C})^{*}: \bigodot \in R \backslash F^{*}\right\}$ so (since $\mathcal{R} \backslash F^{*}$ is compact) pick a finite

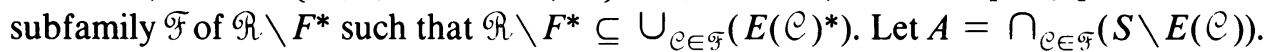
Then $A \in \mathbb{Q}$ and if $\mathscr{Q} \in \mathcal{R} \backslash F^{*}$, then for some $\mathcal{C} \in \mathcal{F}, E(\mathcal{C}) \in \mathscr{\mathcal { L }}$ and hence $S \backslash A \in \mathscr{D}$. Similarly, we obtain $B \in \mathscr{B}$ such that whenever $\mathscr{D} \in \mathscr{R} \backslash G^{*}, S \backslash B \in \mathscr{Q}$. Since $F^{*} \cap G^{*}=\varnothing$ we have that each $\mathscr{Q} \in R$ is either in $R \backslash F^{*}$ or $\mathscr{R} \backslash G^{*}$, and hence $(b)$ is established.

(Sufficiency) Assume that (a) and (b) hold and observe that statement (b) of Theorem 5.2 follows. Thus letting $R=\{(p, q): p, q \in \beta S$ and there is some $Q \in G$ such that $Q \subseteq p \cap q\}$, we have as in Theorem 5.2 that $\mathscr{R}=\left\{\cap[p]_{R}: p \in \beta S\right\}$. To complete the proof it suffices to show that $R$ is closed in $\beta S \times \beta S$. (For then, since $\beta S$ is a compact Hausdorff space, $\beta S / R$ is Hausdorff and hence Lemma 5.5 applies.) To this end let $(p, q) \in(\beta S \times \beta S) \backslash R$. Pick, by Theorem $5.2\left(\mathrm{a}^{\prime}\right), \mathcal{Q}$ and $\mathscr{B}$ in $\mathscr{R}$ such that $Q \subseteq p$ and $\mathscr{Q} \subseteq q$. Since $(p, q) \notin R, \mathbb{Q} \neq \mathscr{R}$. Pick $A \in \mathbb{Q}$ and $B \in \mathscr{B}$ such that either $S \backslash A \in \mathcal{C}$ or $S \backslash B \in \mathcal{C}$ whenever $\mathcal{C} \in R$. Then $\bar{A} \times \bar{B}$ is a neighborhood of $(p, q)$ in $\beta S \times \beta S$ which misses $R$. (If $(u, v) \in \bar{A} \times \bar{B}$ and $\bigodot \in \mathcal{R}$ with $\mathcal{C} \subseteq u \cap v$ and, say $S \backslash A \in \mathcal{C}$, then $S \backslash A \in u$.)

Because of Theorem 5.6 we are justified in making the following definition.

5.7 Definition. $R$ is a quotient of $\beta S$ if and only if $R$ is a set of filters on $\beta S$ with the quotient topology satisfying statements (a) and (b) of Theorem 5.6.

If $R$ is a quotient of $\beta S$, then by Theorem 5.2, for each $p \in \beta S$ there is a unique $Q \in G$ such that $Q \subseteq p$. Consequently, the function $\pi$ below is well defined.

5.8 Definition. Let $R$ be a quotient of $\beta S$. Define $\pi: \beta S \rightarrow$ R by $\pi(p) \subseteq p$. Define $e: S \rightarrow$ G by $e(s)=\pi(\hat{s})$.

We observe that if $\{\hat{s}: s \in S\} \subseteq \Re R$, then for each $s \in S, e(s)=\hat{s}$.

The next result is a corollary to Lemma 5.5 and Theorem 5.6.

\subsection{COROllary. Let R be a quotient of $\beta S$. Then $\pi$ is a quotient map.}

Proof. Let $R, f: \beta S / R \rightarrow \stackrel{R}{R}$, and $g: \beta S \rightarrow \beta S / R$ be as in the proof of Lemma 5.5. By Theorem 5.6, $\beta S / R$ is Hausdorff. Thus $f$ is a homeomorphism. Since $g$ is a quotient map and $\pi=f \circ g$ (given $p \in \beta S, f(g(p))=f([p])=\bigcap[p] \subseteq p), \pi$ is a quotient map.

We need to distinguish now between two notions of compactification. The unmodified word "compactification" will mean a compactification in the sense of general topology. 
5.10 Definition. Let $X$ and $Y$ be topological spaces. The pair $(Y, h)$ is a weak compactification of $X$ if and only if $Y$ is compact, $h: X \rightarrow Y, h$ is continuous, and $h[X]$ is dense in $Y$.

Thus a weak compactification $(Y, h)$ is a compactification if and only if $h$ is an embedding. The term "compactification" is used for a weak compactification in many of the references listed.

A set $R$ of filters may be a compactification of $S$ in many unnatural ways. For example, let $D$ be any countable discrete subset of $\beta \mathbf{N} \backslash \mathbf{N}$. Then $\operatorname{cl}_{\beta \mathbf{N}} D$ is homeomorphic to $\beta \mathbf{N}[7,6.10]$. Thus $\mathrm{cl}_{\beta \mathbf{N}} D$ is a set of (ultra-) filters with quotient topology which is a compactification of $\mathbf{N}$. To avoid this kind of pathology we require in Theorem 5.11 and Corollary 5.12 that each $s \in S$ be an element of every member of the filter with which it is associated. Even then, not all pathologies are eliminated. Consider $R=(\beta N \backslash\{\hat{1}, \hat{2}, \hat{3}\}) \cup\{\hat{1} \cap \hat{2}, \hat{1} \cap \hat{3}, \hat{2} \cap \hat{3}\}$. Then $R$, with the quotient topology, is a compactification of $\mathbf{N}$ under two distinct embeddings which satisfy the above requirement. (One may send $1 \rightarrow \hat{1} \cap \hat{2}, 2 \rightarrow \hat{2} \cap \hat{3}$ and $3 \rightarrow \hat{1} \cap \hat{3}$ or one may send $1 \rightarrow \hat{1} \cap \hat{3}, 2 \rightarrow \hat{1} \cap \hat{2}$ and $3 \rightarrow \hat{2} \cap \hat{3}$.) Accordingly we also require that $\Re$ satisfy condition (b) of Theorem 5.2. (That these two requirements suffice to eliminate all pathologies is part of the content of the next theorem.)

5.11 THEOREM. Let $R$ be a set of filters on $S$ with the quotient topology. The following statements are equivalent.

(a) There exists $h: S \rightarrow \Re$ such that $(\Re, h)$ is a Hausdorff weak compactification of $S$ and

(i) for each $s \in S, s \in \cap h(s)$ and

(ii) for distinct $\mathbb{Q}$ and $\mathscr{B}$ in $\Re$, there exists $B \in \mathscr{B}$ such that $S \backslash B \in \mathbb{Q}$.

(b) $\Re$ is a quotient of $\beta S$.

(c) $\Re$ is a quotient of $\beta S,(\Re, e)$ is a Hausdorff weak compactification of $S$, and $e$ is an open map.

Proof. To see that (a) implies (b), we must show that conditions (a) and (b) of Theorem 5.6 hold. Let $f$ be a choice function for $\mathscr{R}$. Then $\left\{f(\mathbb{Q})^{*}: \mathbb{Q} \in \mathcal{R}\right\}$ is an open cover of $R$ so pick finite $\mathscr{F} \subseteq R$ such that $R \subseteq \cup_{\mathscr{Q} \in \mathscr{F}} f(\mathbb{Q})^{*}$. To see that $S \subseteq \cup_{\mathscr{Q} \in \mathscr{F}} f(\mathbb{Q})$, let $s \in S$. Pick $\mathbb{Q} \in \mathcal{F}$ such that $h(s) \in f(\mathbb{Q})^{*}$. Then $f(\mathbb{Q}) \in h(s)$ and $s \in \cap h(s)$ so $s \in f(\mathbb{Q})$.

The proof that condition (b) of Theorem 5.6 holds can be taken nearly verbatim from the proof of Theorem 5.6.

To see that (b) implies (c) observe that by Theorem 5.6, $R$ is a compact Hausdorff space. Since $S$ is discrete, $e$ is continuous. To see that $e[S]$ is dense in $\Re$, let $A \subseteq S$ such that $A^{*} \neq \varnothing$ and pick $\mathbb{Q} \in \mathcal{R}$ such that $\mathbb{Q} \in A^{*}$. Pick $p \in \beta S$ such that $\mathbb{Q} \subseteq p$. Then $\pi(p)=\mathcal{Q}$ so $\pi(p) \in A^{*}$ so pick $B \in p$ such that $\pi[\bar{B}] \subseteq A^{*}$. (By Corollary 5.9, $\pi$ is continuous.) Pick $s \in B$. Then $\hat{s} \in \bar{B}$ so $e(s)=\pi(\hat{s}) \in A^{*}$. To see that $e$ is open observe that $e$ is the composition of the quotient map $\pi$ with the open embedding of $S$ into $\beta S$.

That (c) implies (a) is trivial. 
5.12 Corollary. Let $\Re$ be a set of filters on $S$ with the quotient topology. The following statements are equivalent.

(a) There exists $h: S \rightarrow \Re$ such that $(\Re, h)$ is a Hausdorff compactification of $S$ and

(i) for each $s \in S, s \in \cap h(s)$ and

(ii) for distinct $\mathscr{Q}$ and $\mathscr{B}$ in $\mathcal{R}$ there exists $B \in \mathscr{B}$ such that $S \backslash B \in \mathbb{Q}$.

(b) $R$ is a quotient of $\beta S$ and $\{\hat{s}: s \in S\} \subseteq \Re$.

(c) $R$ is a quotient of $\beta S$ and $(\Re, e)$ is a Hausdorff compactification of $S$.

Proof. To see that (a) implies (b) we need only show that $\{\hat{s}: s \in S\} \subseteq \Re$. (The rest of the statement follows from Theorem 5.11.) Let $s \in S$. We show that $\{s\} \in h(s)$ and hence that $h(s)=\hat{s}$. Suppose instead that $\{s\} \notin h(s)$ so that $h(s) \cup\{S \backslash\{s\}\}$ has the finite intersection property. Pick $p \in \beta S$ such that $h(s) \cup$ $\{S \backslash\{s\}\} \subseteq p$.

We claim that $h^{\beta}(p) \subseteq p$. To this end let $A \in h^{\beta}(p)$ and, since $h^{\beta}$ is continuous and $h^{\beta}(p) \in A^{*}$, pick $B \in p$ such that $h^{\beta}[\bar{B}] \subseteq A^{*}$. Now given $t \in B, h(t)=h^{\beta}(\hat{t})$ $\in A^{*}$ so $A \in h(t)$ and hence $t \in A$ by (i). Thus $B \subseteq A$ so $A \in p$, as required.

Now $h(s) \subseteq p$ and $h^{\beta}(p) \subseteq p$ so by Theorem 5.2 (using (ii)) we have $h^{\beta}(p)=h(s)$.

Since $h[S]$ is homeomorphic with $S$ it is locally compact. Thus, since $h[S]$ is dense in $\mathcal{R}, h[S]$ is open in $\Re$ and hence $\{h(s)\}$ is open in $R$. Pick $D \in h(s)$ such that $D^{*}=\{h(s)\}$. Since $h^{\beta}(p) \in D^{*}$, pick $C \in p$ such that $h^{\beta}[\bar{C}] \subseteq D^{*}$. Pick $x \in C \cap$ $(S \backslash\{s\})$. Then $\hat{x} \in \bar{C}$ so $h(x)=h^{\beta}(\hat{x}) \in D^{*}=\{h(s)\}$. Thus $h(x)=h(s)$, contradicting the fact that $h$ is one-to-one.

To see that (b) implies (c) we need only show by Theorem 5.11 that $e$ is one-to-one. Since for each $s \in S, e(s)=\hat{s}$, this is trivial.

That (c) implies (a) is trivial.

We now turn our attention to extending the semigroup structure of $S$ to $R$. We assume that $(S,+)$ is a discrete semigroup. We first have need of the following characterization of + on $\beta S$, which motivates our definition of + on $R$. This characterization is due to $\mathrm{S}$. Glazer (see [5 or 11]).

5.13 Theorem (Glazer). Let $p, q \in \beta S$ and let $A \subseteq S$. Then $A \in p+q$ if and only if $\{s \in S: A-s \in p\} \in q$.

Proof. (Necessity) Since $\lambda_{p}(q) \in \bar{A}$, pick $B \in q$ such that $\lambda_{p}[\bar{B}] \subseteq \bar{A}$. For each $s \in B, p+s \in \bar{A}$ so, by Lemma $2.1, A-s \in p$. Thus $B \subseteq\{s \in S: A-s \in p\}$.

(Sufficiency) Suppose $A \notin p+q$. Then $S \backslash A \in p+q$ so $\{s \in S:(S \backslash A)-s \in$ $p\} \in q$ while for any $s \in S,((S \backslash A)-s) \cap(A-s)=\varnothing$, a contradiction.

5.14 Definition. Let $\mathcal{Q}$ and $\mathscr{B}$ be filters on $S$. Then

$$
\mathscr{Q}+\mathscr{B}=\{A \subseteq S:\{s \in S: A-s \in \mathbb{Q}\} \in \mathscr{B}\}
$$

5.15 Lemma. Let $\mathscr{Q}, \mathscr{B}$ and $\mathcal{C}$ be filters on $S$. Then:

(1) $Q+\mathscr{B}$ is a filter on $S$.

(2) $(Q+\Re)+C=Q+(\mathscr{Q}+\mathcal{C})$.

(3) $\bar{Q}+\overline{\mathscr{B}} \subseteq \mathrm{cl}_{\beta S}(\bar{Q}+\overline{\mathscr{Q}}) \subseteq \overline{Q+\mathscr{G}}$

and there exist filters $\mathcal{Q}$ and $\Re$ on $\mathbf{N}$ such that both inclusions are proper. 
Proof. $S=\{s \in S: S-s \in \mathbb{Q}\}$ and $\varnothing=\{s \in S: \varnothing-s \in \mathbb{Q}\}$, so $S \in \mathbb{Q}+\mathscr{B}$ and $\varnothing \notin \mathbb{Q}+\mathscr{B}$. If $A \in \mathbb{Q}+\mathscr{B}$ and $A \subseteq B \subseteq S$, then $\{s \in S: A-s \in \mathbb{Q}\} \subseteq\{s \in S$ : $B-s \in \mathbb{Q}\}$, so $B \in \mathbb{Q}+\mathbb{B}$. If $A, B \in \mathbb{Q}+\mathscr{B}$, then $\{s \in S: A-s \in \mathbb{Q}\} \cap\{s \in S$ : $B-s \in \mathbb{Q}\} \subseteq\{s \in S:(A \cap B)-s \in \mathbb{Q}\}$; so $A \cap B \in \mathbb{Q}+\mathbb{B}$. Thus (1) holds.

To verify (2), note first that for $A \subseteq S$ and $s$ and $t$ in $S$ one has $A-(t+s)=(A$ $-s)-t$. Thus, given $s \in S$ and $A \subseteq S$, we have $\{x \in S: A-x \in \mathbb{Q}\}-s=\{t \in S$ : $(A-s)-t \in \mathbb{Q}\}$. Thus for $A \subseteq S$, we have

$$
\begin{aligned}
A \in \mathbb{Q}+(\mathscr{B}+\mathcal{C}) & \Leftrightarrow\{x \in S: A-x \in \mathbb{Q}\} \in \mathscr{B}+\mathcal{C} \\
& \Leftrightarrow\{s \in S:\{x \in S: A-x \in \mathbb{Q}\}-s \in \mathscr{B}\} \in \mathcal{C} \\
& \Leftrightarrow\{s \in S:\{t \in S:(A-s)-t \in \mathbb{Q}\} \in \mathscr{B}\} \in \mathcal{C} \\
& \Leftrightarrow\{s \in S: A-s \in \mathbb{Q}+\mathscr{B}\} \in \mathcal{C} .
\end{aligned}
$$

The first inclusion of $(3)$ is trivial. For the second, since $\overline{\mathbb{Q}+\mathscr{T}}$ is closed in $\beta S$ it suffices to show $\overline{\mathbb{Q}}+\overline{\mathscr{B}} \subseteq \overline{\mathbb{Q}+\mathscr{B}}$. To this end let $p \in \overline{\mathbb{Q}}$ and let $q \in \overline{\mathscr{B}}$. Let $A \in \mathbb{Q}+\mathscr{B}$. Then $\{s \in S: A-s \in \mathbb{Q}\} \in q$ and $\{s \in S: A-s \in \mathbb{Q}\} \subseteq\{s \in S$ : $A-s \in p\}$ so $A \in p+q$.

To see that the inclusions of (3) may be proper, let $Q=\{\mathbf{N}\}$, pick $p$ in the minimal ideal of $(\beta \mathbf{N},+)$ and let $\mathscr{B}=p$. Then $\overline{\mathbb{Q}}=\beta \mathbf{N}$ and $\bar{p}=p$ while, by Theorem 7.16 of [10], $\beta \mathbf{N}+p$ is not closed. Thus $\overline{\mathscr{Q}}+\overline{\mathscr{B}} \neq \mathrm{cl}_{\beta \mathbf{N}}(\overline{\mathbb{Q}}+\overline{\mathscr{B}})$. Also by Theorem 7.12 of [10], $(\beta \mathbf{N} \backslash \mathbf{N},+)$ has a proper closed ideal $I$ and $\beta \mathbf{N}+p$ is contained in the minimal ideal so $\operatorname{cl}(\overline{\mathbb{Q}}+\overline{\mathscr{B}}) \subseteq I \subsetneq \beta \mathbf{N} \backslash \mathbf{N}$. To complete the proof we show $\beta \mathbf{N} \backslash \mathbf{N}$ $=\overline{Q+G}$. No singleton is in $Q+\mathscr{B}$ so $\bar{Q}+\mathscr{B} \subseteq \beta \mathbf{N} \backslash \mathbf{N}$. Now let $q \in \beta \mathbf{N} \backslash \mathbf{N}$ and let $A \in \mathbb{Q}+\mathscr{B}$. Then $\{n \in \mathbf{N}: A-n \in \mathbb{Q}\} \in \mathscr{R}$ so pick $n \in \mathbf{N}$ such that $A-n \in \mathbb{Q}$. Then $A-n=\mathbf{N}$ so $A$ is cofinite so $A \in q$.

5.16 Definition. Let $\mathcal{R}$ be a quotient of $\beta S$. If for all $Q$ and $\mathscr{B}$ in $\mathcal{R}$ there is some

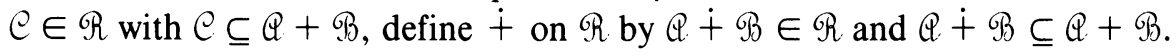

5.17 Lemma. Let $(\Re, \dot{+})$ be as in Definition 5.16. Then the diagram below commutes:

$$
\begin{array}{ccc}
\beta S \times \beta S & \stackrel{+}{\rightarrow} & \beta S \\
\downarrow \pi \times \pi & & \downarrow \pi \\
\mathscr{G} \times \mathscr{G} & \stackrel{+}{\rightarrow} & \mathscr{R}
\end{array}
$$

Proof. We first observe that $\dot{+}$ is well defined. Indeed, if $\mathcal{C}$ and $\mathscr{O})$ are in $R$ and

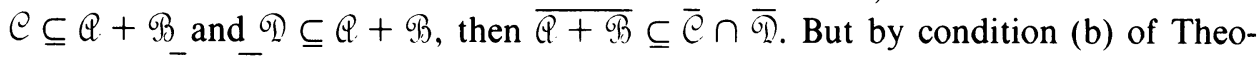
rem 5.6, if $\bar{\complement} \cap \bar{\Phi} \neq \varnothing$, then $\circlearrowright=\mathscr{Q}$.

Now let $p$ and $q$ be in $\beta S$. Let $Q=\pi(p), \mathscr{Q}=\pi(q), \mathcal{C}=\pi(p+q)$, and $Q \mathcal{Q})=$ $\pi(p)+\pi(q)$. Now $p+q \in \overline{\mathbb{Q}}+\overline{\mathscr{B}}$ so by Lemma $5.15, p+q \in \overline{\mathbb{P}+\mathscr{B}}$. Since

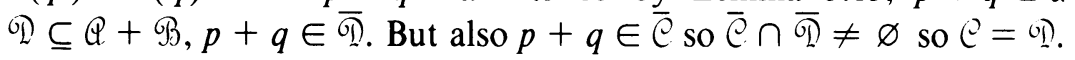

5.18 THEOREM. Let $(\Re, \dot{+})$ be as in Definition 5.16. Then

(1) $e$ is a (continuous) homomorphism from $S$ to $R$,

(2) $R$ is a left topological semigroup,

(3) $e[S]$ is dense in $R$, and

(4) for every $s \in S$, the function $\rho_{e(s)}$ is continuous. 
Proof. Let $s, t \in S$. It is routine to verify that $\hat{s}+\hat{t}=(s+t) \hat{)}$. Also by Lemma 5.17, we have $\pi(\hat{s}) \dot{+} \pi(\hat{t})=\pi(s+t)$. Thus $e(s+t)=\pi((s+t) \hat{)})=\pi(\hat{s}+\hat{t})=$ $\pi(\hat{s}) \dot{+} \pi(\hat{t})=e(s)+e(t)$. Thus $e$ is a (necessarily continuous) homomorphism.

That $\dot{t}$ is associative follows from Lemma 5.15(2), using the fact that for any

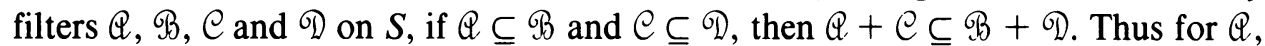
$\mathscr{B}$ and $C$ in $R,(Q+\mathscr{B})+C \subseteq(Q+\mathscr{B})+C \subseteq(Q+\mathscr{B})+C$ and $Q+(\mathscr{B}+C) \subseteq$ $Q+(\mathscr{B}+\mathcal{C}) \subseteq Q+(\mathscr{Q}+\mathcal{C})$. Therefore $\overline{(Q+\mathscr{+} \mathscr{B}) \dot{+} \mathcal{C}} \cap \overline{Q \dot{Q}+(\mathscr{B}+\mathcal{C})} \neq \varnothing$ so $(Q+B)+C=Q+(\mathscr{C}+C)$.

To see that $\dot{+}$ is left continuous, let $Q \in R$ and pick $p \in \bar{Q}=\pi^{-1}[\{\mathscr{Q}\}]$. By Lemma 5.17, we have $\lambda_{\mathscr{Q}} \circ \pi=\pi \circ \lambda_{p}$. By Theorem 2.2, $\lambda_{p}$ is continuous and by Corollary 5.9, $\pi$ is a quotient map. Thus $\lambda_{\mathscr{Q}}$ is continuous.

By Theorem 5.11,e[S] is dense in $R$. Finally, let $s \in S$. Then by Lemma 5.17, $\rho_{e(s)} \circ \pi=\pi \circ \rho_{\hat{s}}$. Since $\rho_{\hat{s}}$ is continuous and $\pi$ is a quotient map, $\rho_{e(s)}$ is continuous.

Given that Theorem 5.18 was our hoped for result, two questions arise about the definition of $\dot{+}$ on $\Re$. The first is whether the requirement that, for each $\mathscr{Q}$ and $\mathscr{B}$ in $\Re$ there be some $\mathcal{C}$ in $\Re$ with $\mathcal{C} \subseteq \mathbb{Q}+\mathscr{G}$, was necessary. The second is whether one could, in fact, take $\dot{+}$ equal to + . That is, could one require $Q+\Re \in R$ whenever $Q$ and $\mathscr{B}$ are in $R$ ? (Evidence in favor of the latter question comes from the fact that it is true when $\Re=\beta S$.) Theorem 5.19 answers the first question in the affirmative, at least when $R$ is a topological compactification of $S$, and Theorem 6.19 answers the second question in the negative.

5.19 Theorem. Let $\Re$ be a quotient of $\beta S$. Assume that $(\Re, e)$ is a Hausdorff compactification of $S$ and that $(\mathcal{R}, *)$ satisfies Theorem 5.18 with $*$ replacing $\dot{+}$. Then whenever $\mathbb{Q}$ and $\mathscr{B}$ are in $\mathscr{R}, \mathbb{Q} * \mathscr{B} \subseteq \mathbb{Q}+\mathfrak{B}$, so that $*$ equals $\dot{+}$.

Proof. Since $(\Re, e)$ is a compactification of $\beta S$, we have by Corollary 5.12 that for $s \in S, e(s)=\hat{s}$. Let $\mathbb{Q} \in \mathcal{R}$ and let $s \in S$. We show first that $\mathbb{Q} * \hat{s} \subseteq \mathbb{Q}+\hat{s}$. Let $A \in \mathbb{Q} * \hat{s}$. Then $A^{*}$ is a neighborhood of $\mathcal{Q} * \hat{s}$ in $\Re$ so pick a neighborhood $B^{*}$ of $\mathbb{Q}$ in $\Re$ such that $\rho_{\hat{s}}\left[B^{*}\right] \subseteq A^{*}$. Then, if $t \in B, \hat{t} * \hat{s} \in A^{*}$. Since $e$ is a homomorphism, $\hat{t} * \hat{s}=(t+s)^{\hat{s}}$ so that $t+s \in A$, i.e. $t \in A-s$. Therefore $B \subseteq A-s$ so $A-s \in \mathbb{Q}$. Thus $s \in\{t \in S: A-t \in \mathbb{Q}\}$ so $A \in \mathbb{Q}+\hat{s}$ as desired.

Now let $\mathbb{Q}$ and $\mathscr{B}$ be in $R$ and let $A \in \mathbb{Q} * \mathscr{B}$. Since $A^{*}$ is a neighborhood of $\mathscr{Q} * \mathscr{B}$, pick $B \in \mathscr{B}$ such that $\lambda_{\mathscr{Q}}\left[B^{*}\right] \subseteq A^{*}$. We then have that for all $s \in B, A \in \mathbb{Q} * \hat{s}$ so that $A \in \mathbb{Q}+\hat{s}$ and hence $A-s \in \mathbb{Q}$. Thus $B \subseteq\{s \in S: A-s \in \mathbb{Q}\}$ so that $A \in \mathbb{Q}+\mathscr{B}$, as desired.

6. The weak almost periodic compactification as a space of filters. In this section we assume throughout that $S$ is a discrete topological semigroup. Our main objective is to describe $\omega S$ as a space of filters. We obtain, in Theorem 6.13, a description of $\omega S$ as a space of filters which is internal to the set of filters. This description turns out to be useful at once in yielding new information about $\omega \mathbf{N}$.

6.1 LEMma. Let $\Re$ be a quotient of $\beta S$. Let $T$ be a compact Hausdorff space, and let $f: S \rightarrow T$. Statements (a) and (d) are equivalent. If $\{\hat{s}: s \in S\} \subseteq \mathcal{R}$, then all four statements are equivalent. 
(a) $f$ has a continuous extension to $\mathscr{R}$. (That is, there exists $g: \Re \rightarrow T$ such that $g \circ e=f$.)

(b) For each $\mathbb{Q} \in \mathscr{R}, \cap\left\{\mathrm{cl}_{T}(f[A]): S \backslash A \notin \mathbb{Q}\right\} \neq \varnothing$.

(c) For each $\mathbb{Q} \in \mathcal{R}, \operatorname{card}\left(\cap\left\{\operatorname{cl}_{T}(f[A]): S \backslash A \notin \mathbb{Q}\right\}\right)=1$.

(d) For each $\mathbb{A} \in \mathcal{R}, f^{\beta}$ is constant on $\overline{\mathcal{Q}}$.

Proof. To see that (a) implies (d), note that $g \circ \pi$ is a continuous extension of $f$ and hence $g \circ \pi=f^{\beta}$.

To see that (d) implies (a), define $g$ by $g(\pi(p))=f^{\beta}(p)$. Since $f$ is constant on $\pi^{-1}[\mathcal{Q}]$ for each $\mathbb{Q} \in \mathcal{R}, g$ is well defined. Since $g \circ \pi=f^{\beta}$ and $\pi$ is a quotient map, $g$ is continuous.

Now assume that $\{\hat{s}: s \in S\} \subseteq \Re$ so that for all $s \in S, e(s)=\hat{s}$. That (c) implies (b) is trivial. To see that (a) implies (c), we show that $\cap\left\{\operatorname{cl}_{T}(f[A]): S \backslash A \notin \mathbb{Q}\right\}=$ $\{g(\mathbb{Q})\}$. First let $A \subseteq S$ such that $S \backslash A \notin \mathbb{Q}$, and suppose $g(\mathbb{Q}) \notin \operatorname{cl}_{T}(f[A])$. Pick a neighborhood $U$ of $g(\mathbb{Q})$ in $T$ such that $U \cap f[A]=\varnothing$. Pick $B \in \mathbb{Q}$ such that $g\left[B^{*}\right] \subseteq U$. Since $S \backslash A \notin \mathbb{Q}$, we get $B \cap A \neq \varnothing$ so pick $s \in B \cap A$. Then $f(s)=$ $g(\hat{s}) \in U$ while $f(s) \in f[A]$. Now suppose $t \in \cap\left\{\operatorname{cl}_{T}(f[A]): S \backslash A \notin \mathbb{Q}\right\}$ and $t \neq g(\mathbb{Q})$. Pick disjoint neighborhoods $U$ and $V$ of $t$ and $g(\mathbb{Q})$, respectively, in $T$. Pick $A \in \mathbb{Q}$ such that $g\left[A^{*}\right] \subseteq V$. Since $S \backslash A \notin \mathbb{Q}$, pick $z \in U \cap f[A]$ and pick $x \in A$ such that $f(x)=z$. But then $z=g(\hat{x}) \in V$, a contradiction.

To see that (b) implies (a), choose for each $\mathscr{Q} \in \mathcal{R}$ some $g(\mathbb{Q}) \in \cap\left\{\operatorname{cl}_{T}(f[A])\right.$ : $S \backslash A \notin \mathbb{Q}\}$. To see that $g$ is continuous, let $\mathcal{Q} \in \mathcal{R}$ and let $U$ be a neighborhood of $g(\mathbb{Q})$ in $T$. Pick a neighborhood $V$ of $g(\mathscr{Q})$ in $T$ such that $\operatorname{cl} V \subseteq U$. Let $A=f^{-1}[V]$. Since $g(\mathbb{Q}) \notin \operatorname{cl}_{T}(f[S \backslash A])$, we have $A \in \mathcal{Q}$. To see that $g\left[A^{*}\right] \subseteq U$, let $\Re \in A^{*}$ and suppose $g(\Re) \notin U$. Then $T \backslash \mathrm{cl} V$ is a neighborhood of $g(\Re)$ and $A \in \mathscr{B}$ so $(T \backslash \operatorname{cl} V) \cap f[A] \neq \varnothing$, a contradiction. Trivially $g \circ e=f$.

6.2 LEMmA. Let $\Re$ be a quotient of $\beta S$ such that $\{\hat{s}: s \in S\} \subseteq \Re$, let $(T,+)$ be a compact Hausdorff left topological semigroup and let f be a homomorphism from $S$ to $T$ such that $\rho_{f(x)}$ is continuous for each $x \in S$. If for each $\mathscr{Q} \in \mathscr{R}, \cap\left\{\operatorname{cl}_{T}(f[A])\right.$ : $S \backslash A \notin \mathbb{Q}\} \neq \varnothing$ and for each $\mathbb{Q}$ and $\mathscr{T}$ in $\mathscr{R}, \mathbb{Q}+\mathscr{B} \in \mathfrak{R}$, then there is a continuous homomorphism $\mathrm{g}: \mathcal{R} \rightarrow T$ such that $\mathrm{g} \circ e=f$.

Proof. We need only prove that $g$ as constructed in Lemma 6.1 is a homomorphism. To this end, let $\mathscr{Q}, \mathscr{B} \in \mathcal{R}$ and suppose $g(\mathscr{Q}+\mathscr{B}) \neq g(\mathscr{Q})+g(\mathscr{B})$. Then $g(\mathbb{Q})+g(\Re) \notin \cap\left\{\operatorname{cl}_{T}(f[A]): S \backslash A \notin \mathbb{Q}+\Re\right\}$. Pick $D \subseteq S$ such that $S \backslash D \notin \mathbb{Q}$ $+\Re$ and $g(\mathscr{Q})+g(\Re) \notin \operatorname{cl}_{T}(f[D])$. Pick a neighborhood $U$ of $g(\mathscr{Q})+g(\Re)$ such that $U \cap f[D]=\varnothing$, pick a neighborhood $V$ of $g(\Re)$ such that $g(\mathscr{Q})+V \subseteq U$ and pick $B \in \mathscr{B}$ such that $g\left[B^{*}\right] \subseteq V$. Now $S \backslash D \notin \mathbb{Q}+\mathscr{B}$ so $B \cap\{x \in S:(S \backslash D)-x$ $\notin \mathbb{Q}\} \neq \varnothing$. Pick $x \in B$ such that $(S \backslash D)-x \notin \mathbb{Q}$. Since $x \in B, f(x)=g(\hat{x}) \in V$ so $g(\mathbb{Q})+f(x) \in U$. Since $\rho_{f(x)}$ is continuous, pick a neighborhood $W$ of $g(\mathbb{Q})$ such that $W+f(x) \subseteq U$. Pick $A \in \mathbb{Q}$ such that $g\left[A^{*}\right] \subseteq W$. Since $(S \backslash D)-x \notin \mathbb{Q}$, $A \cap(D-x) \neq \varnothing$. Pick $y \in A \cap(D-x)$. Then $y \in A$ so $f(y)=g(\hat{y}) \in W$. Therefore $f(y)+f(x) \in U$. But $y+x \in D$ so $f(y+x) \in f[D]$ so $f[D] \cap U \neq \varnothing$, a contradiction. 
The notion of $W$-nest, defined below, is the last link in the internal description of $\omega S$ as a set of filters. We use the set $\mathbf{Q}^{+}$of positive rationals in the definition, but any other dense subset of the positive reals would do.

6.3 Definition. (a) $\phi$ is a $W$-nest in $S$ if and only if

(1) $\phi: \mathbf{Q}^{+} \rightarrow \mathscr{P}(S) \backslash\{\varnothing\}$,

(2) $S \in \operatorname{Range}(\phi)$,

(3) if $r, s \in \mathbf{Q}^{+}$and $r<s$, then $\phi(r) \subseteq \phi(s)$, and

(4) there do not exist $r$ and $v$ in $\mathbf{Q}^{+}$and sequences $\left\langle t_{n}\right\rangle_{n=1}^{\infty}$ and $\left\langle s_{n}\right\rangle_{n=1}^{\infty}$ in $S$ such that $r<v$ and whenever $k<n, t_{n}+s_{k} \in \phi(r)$ and $t_{k}+s_{n} \notin \phi(v)$.

(b) $\mathscr{Q}(S)=\{\phi: \phi$ is $W$-nest in $S\}$.

6.4 LEMma. (a) If $f \in W(S), a \in \operatorname{cl}_{\mathbf{C}} f[S]$, and for $r \in \mathbf{Q}^{+}, \phi(r)=\{s \in S$ : $|f(s)-a|<r\}$, then $\phi \in \mathcal{Q}(S)$.

(b) If $\phi \in \mathcal{Q}\left(S(S)\right.$ and $f$ is defined on $S$ by $f(s)=\inf \left\{r \in \mathbf{Q}^{+}: s \in \phi(r)\right\}$, then $f \in W(S)$. Further, if $p \in \beta S$ and $\operatorname{Range}(\phi) \subseteq p$, then $f^{\beta}(p)=0$.

Proof. (a) Conditions (1) and (3) are immediate and (2) holds since $f$ is bounded. To see that (4) holds, suppose we have such $r, v,\left\langle t_{n}\right\rangle_{n=1}^{\infty}$ and $\left\langle s_{n}\right\rangle_{n=1}^{\infty}$. Thin the sequences $\left\langle t_{n}\right\rangle_{n=1}^{\infty}$ and $\left\langle s_{n}\right\rangle_{n=1}^{\infty}$ so that $\lim _{n \rightarrow \infty} \lim _{k \rightarrow \infty} f\left(t_{n}+s_{k}\right)$ and $\lim _{k \rightarrow \infty} \lim _{n \rightarrow \infty} f\left(t_{n}+s_{k}\right)$ exist. Then $\lim _{n \rightarrow \infty} \lim _{k \rightarrow \infty}\left|f\left(t_{n}+s_{k}\right)-a\right| \geqslant v$ while $\lim _{k \rightarrow \infty} \lim _{n \rightarrow \infty}\left|f\left(t_{n}+s_{k}\right)-a\right| \leqslant r$, a contradiction.

(b) By condition (2), $f$ is defined everywhere and is bounded. Suppose $f$ is not weak almost periodic and pick sequences $\left\langle t_{n}\right\rangle_{n=1}^{\infty}$ and $\left\langle s_{n}\right\rangle_{n=1}^{\infty}$ such that $\lim _{n \rightarrow \infty} \lim _{k \rightarrow \infty} f\left(t_{n}+s_{k}\right)=x$ and $\lim _{k \rightarrow \infty} \lim _{n \rightarrow \infty} f\left(t_{n}+s_{k}\right)=y$ with $x>y$. Let $\varepsilon=(x-y) / 3$. By thinning the sequences we may assume that whenever $k<n$, $f\left(t_{n}+s_{k}\right)<y+\varepsilon$ and $f\left(t_{k}+s_{n}\right)>x-\varepsilon$. Pick $r<v$ in $\mathbf{Q}^{+}$such that $y+\varepsilon<r<v$ $<x-\varepsilon$. Then for $k<n, t_{n}+s_{k} \in \phi(r)$ and $t_{k}+s_{n} \notin \phi(v)$.

The last conclusion holds since each neighborhood of $p$ includes points $\hat{s}$ with $f(s)$ arbitrarily close to 0 .

6.5 Definition. Define an equivalence relation on $\beta S$ by $p \sim q$ if and only if $f^{\beta}(p)=f^{\beta}(q)$ whenever $f \in W(S)$.

We observe that $\sim$ is trivially an equivalence relation on $\beta S$. We shall be interested in the filters $\cap[p]_{\sim}$ where $p \in \beta S$. Consequently, we want to know when $A \in \cap[p]_{\sim}$ in terms of $p$.

6.6 Lemma. Let $p \in \beta S$ and let $A \subseteq S$. The following statements are equivalent.

(a) $A \in \cap[p]_{\sim}$.

(b) There exist $f \in W(S)$ and $\delta>0$ such that $\left\{s \in S:\left|f(s)-f^{\beta}(p)\right|<\delta\right\} \subseteq A$.

(c) There exist $f \in W(S)$ and $\delta>0$ such that $\operatorname{Range}(f) \subseteq\{x \in \mathbf{R}: 0 \leqslant x \leqslant 1\}$, $f^{\beta}(p)=0$, and $f^{-1}[[0, \delta)] \subseteq A$.

Proof. That (c) implies (b) is trivial. To see that (b) implies (a), let $q \in[p]$. Then $f^{\beta}(q)=f^{\beta}(p)$ so $B=\left\{s \in S:\left|f(s)-f^{\beta}(q)\right|<\delta\right\} \subseteq A$. Since $B \in q$, we have $A \in q$.

To see that (a) implies (c), suppose the conclusion fails and let

$$
G=\left\{f \in W(S): \text { Range }(f) \subseteq\{x \in \mathbf{R}: 0 \leqslant x \leqslant 1\}, \text { and } f^{\beta}(p)=0\right\} .
$$


For each $f \in G$ and each $\delta>0$, let $B(f, \delta)=f^{-1}[[0, \delta)] \backslash A$ and let $\mathcal{Q}=\{B(f, \delta)$ : $f \in G$ and $\delta>0\}$. Given $f$ and $g$ in $G, \delta>0$ and $\gamma>0$, let $\mu=\min \{\delta, \gamma\}$ and define $h$ by $h(s)=\max \{f(s), g(s)\}$. Then Range $(h) \subseteq\{x \in \mathbf{R}: 0 \leqslant x \leqslant 1\}$ and, by Lemma $2.8, h \in W(S)$. To see that $h^{\beta}(p)=0$, suppose instead that $h^{\beta}(p)>y>0$. Then $\{s \in S: h(s)>y\} \in p$ and hence either $\{s \in S: f(x)>y\} \in p$ or $\{s \in S: g(s)>y\}$ $\in p$, a contradiction. Thus $h \in G$ and $B(h, \mu) \subseteq B(f, \delta) \cap B(g, \gamma)$. Therefore $Q$ has the finite intersection property. Pick $r \in \beta S$ such that $Q \subseteq r$. Then $A \notin r$ so $r \notin[p]$. Pick $g \in W(S)$ such that $g^{\beta}(p) \neq g^{\beta}(r)$. Let $b=\sup \left\{\left|g^{\beta}(q)-g^{\beta}(p)\right|: q \in \beta S\right\}$ and pick $n \in \mathbf{N}$ such that $n>b$. Define $\phi$ as in Lemma 6.4(a) with $a=g^{\beta}(p)$. Then $\phi \in \mathcal{Q}(S)$ and $\phi(n)=S$. Define $\mu(t)$ for $t \in \mathbf{Q}^{+}$by $\mu(t)=\phi(n t)$. Then trivially $\mu \in \mathcal{Q}(S)$. Define $f$ on $S$ by $f(s)=\inf \left\{t \in \mathbf{Q}^{+}: s \in \mu(t)\right\}$. By Lemma 6.4(b), $f \in W(S)$. Since $\mu(1)=S$, Range $(f) \subseteq\{x \in \mathbf{R}: 0 \leqslant x \leqslant 1\}$. Given $t \in \mathbf{Q}^{+}, \mu(t)$ $=\left\{s \in S:\left|g(s)-g^{\beta}(p)\right|<n t\right\}$ so $\mu(t) \in p$. Thus, again by Lemma 6.4(b), $f^{\beta}(p)$ $=0$. Also $f^{\beta}(r)=\left|g^{\beta}(r)-g^{\beta}(p)\right| / n>0$. Thus $B\left(f, f^{\beta}(r) / 2\right) \in \mathbb{Q} \backslash r$, a contradiction.

The following theorem is the basis for the internal characterization of $\omega S$.

6.7 THEOREM. Let $\Re$ be a set of filters on S. Statement (a) below is equivalent to the conjunction of statements (b), (c) and (d) and implies that ( $(R, e)$ is a Hausdorff weak compactification of $S$.

(a) $R=\left\{\cap[p]_{\sim}: p \in \beta S\right\}$.

(b) For any $\phi \in \mathcal{Q}(S)$ and any $\Theta \in R$, if $\Theta \cup \operatorname{Range}(\phi)$ has the finite intersection property, then $\operatorname{Range}(\phi) \subseteq Q$.

(c) Given distinct $\Theta$ and $\mathscr{Q}$ in $R$, there exists $\phi \in \mathcal{Q}(S)$ such that $\operatorname{Range}(\phi) \subseteq($ but $\operatorname{Range}(\phi) \backslash \mathscr{T} \neq \varnothing$.

(d) For each choice function $f$ for $R$, there is a finite subfamily $\mathscr{F}$ of $R$ such that $S=\cup_{\mathbb{Q} \in \mathcal{F}} f(\mathbb{Q})$.

Proof. We show first that (a) implies (b), (c) and (d), so we assume (a) holds. Observe that if $p, q \in \beta S$ and $p \notin[q]$, then for some $f \in W(S), f^{\beta}(p) \neq f^{\beta}(q)$. Since $f^{\beta}$ is continuous there is a neighborhood of $p$ missing $[q]$. That is, each $[q]$ is closed in $\beta S$. Thus by Theorem 5.2, condition (d) holds.

To establish (b), let $\phi \in \mathcal{Q} \int(S)$ and let $\Theta \in R$ and assume $\mathcal{Q} \cup \operatorname{Range}(\phi)$ has the finite intersection property. Pick $p \in \beta S$ such that $\Theta \cup \operatorname{Range}(\phi) \subseteq p$. Then $(\mathcal{\psi}=$ $\cap[p]$. Suppose Range $(\phi) \backslash \mathscr{Q} \neq \varnothing$ and pick $q \in[p]$ and $r \in \mathbf{Q}^{+}$such that $\phi(r) \notin q$. Define $f$ as in Lemma 6.4(b). Then $f^{\beta}(p)=0$ while $f^{\beta}(q) \geqslant r$, a contradiction.

To establish (c), let $Q$ and $\mathscr{B}$ be distinct members of $Q R$ and pick $p$ and $q$ in $\beta S$ such that $\mathbb{Q}=\cap[p]$ and $\mathscr{B}=\cap[q]$. Pick $f \in W(S)$ such that $f^{\beta}(p) \neq f^{\beta}(q)$ and let $a=f^{\beta}(p)$. Define $\phi$ as in Lemma 6.4(a). Let $\varepsilon=\left|f^{\beta}(q)-f^{\beta}(p)\right|$ and pick $v \in \mathbf{Q}^{+}$ such that $v<\varepsilon$. Then $\phi(v) \notin q$ so $\phi(v) \notin$ भ. Further, if $r \in[p]$, then $f^{\beta}(r)=a$ so $\operatorname{Range}(\phi) \subseteq r$. Therefore Range $(\phi) \subseteq \mathbb{Q}$.

Now we assume that (b), (c) and (d) hold and prove (a). Since (d) holds, by Theorem 5.2 each $p \in \beta S$ contains some $Q \in R$. It suffices, therefore, to show that if $p \in \beta S$ and $Q \in G R$ with $\Theta \subseteq p$, then $\Theta=\cap[p]$. To this end, let $p \in \beta S$ and let $Q \in \mathcal{Q}$ with $\Theta \subseteq p$. Let $q \in[p]$ and suppose $(\mathcal{Q} \backslash q \neq \varnothing$. By condition (d), pick 
$\Re \in \mathscr{R}$ such that $\mathscr{\Re} \subseteq q$. Pick $\phi$ as guaranteed by condition (c). Note that if we had Range $(\phi) \subseteq q$, we would have $\Re \cup \operatorname{Range}(\phi)$ having the finite intersection property and would thus have $\operatorname{Range}(\phi) \subseteq \Re$, by condition (b). Thus we pick $r \in \mathbf{Q}^{+}$such that $\phi(r) \notin q$. Define $f$ as in Lemma 6.4(b). Then $f^{\beta}(q) \geqslant r$ while $f^{\beta}(p)=0$, a contradiction. Thus $\mathscr{Q} \subseteq \cap[p]$. To see that $\cap[p] \subseteq \mathscr{Q}$, let $A \in \cap[p]$ and suppose $A \notin \mathbb{Q}$. Pick $q \in \beta S$ such that $\mathbb{Q} \cup\{S \backslash A\} \subseteq q$ and pick $f \in W(S)$ such that $f^{\beta}(p) \neq f^{\beta}(q)$. Let $a=f^{\beta}(p)$ and define $\phi$ as in Lemma 6.4(a). Then Range $(\phi) \subseteq p$ so $Q \cup \operatorname{Range}(\phi)$ has the finite intersection property so by condition (b), $\operatorname{Range}(\phi)$ $\subseteq \mathcal{Q}$. But then Range $(\phi) \subseteq q$ so $f^{\beta}(p)=f^{\beta}(q)$, a contradiction.

Now assume that (a) holds. To show that $(\Re, e)$ is a Hausdorff weak compactification of $S$ it suffices, by Theorem 5.11, to show that $R$ is a quotient of $\beta S$, that is, that conditions (a) and (b) of Theorem 5.6 hold. Since condition (a) of Theorem 5.6 and condition (d) of this theorem are identical, it suffices to establish condition (b) of Theorem 5.6. To this end, let $\mathcal{Q}$ and $\mathscr{B}$ be distinct members of $\Re$ and pick $p$ and $q$ in $\beta S$ such that $\mathbb{Q}=\bigcap[p]$ and $\mathscr{G}=\bigcap[q]$. Pick $f \in W(S)$ such that $f^{\beta}(p) \neq f^{\beta}(q)$. Let $\varepsilon=\left|f^{\beta}(p)-f^{\beta}(q)\right|$, let $A=\left\{s \in S:\left|f(s)-f^{\beta}(p)\right|<\varepsilon / 3\right\}$ and let $B=\{s \in$ $\left.S:\left|f(s)-f^{\beta}(q)\right|<\varepsilon / 3\right\}$. Then $A \in \mathbb{Q}$ and $B \in \mathscr{G}$. Let $\mathcal{C} \in \Re$ and pick $r \in S$ such that $\mathcal{C}=\cap[r]$. If $\left|f^{\beta}(r)-f^{\beta}(p)\right|<2 \varepsilon / 3$, then $S \backslash B \in \mathcal{C}$ and if $\left|f^{\beta}(r)-f^{\beta}(p)\right|>$

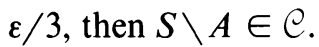

We want to show that $\Re=\left\{\cap[p]_{\sim}: p \in \beta S\right\}$ is $\omega S$. In order to use Theorem

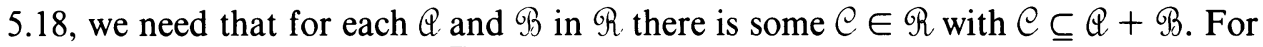
such $\mathcal{C}$, we have $\overline{\mathcal{Q}+\mathscr{G}} \subseteq \overline{\mathcal{C}}$. By Lemma 5.15(3) we have, if $\mathscr{Q}=\cap[p]$ and $\mathscr{B}=\cap[q]$, that $p+q \in \overline{\mathcal{Q}+\mathscr{B}}$. Since for distinct $\mathcal{C}$ and $\mathscr{D}$ in $\Re, \bar{C} \cap \overline{\mathscr{D}}=\varnothing$, our only candidate for $C$ in $R$ with $C \subseteq Q+\mathscr{Q}$ is thus $\cap[p+q]$.

6.8 LEMMA. If for all $p, q$ and $r$ in $\beta S, q \sim r$ implies $p+q \sim p+r$, then for all $p$ and $q$ in $\beta S$,

$$
\cap[p+q]_{\sim} \subseteq \cap[p]_{\sim}+\cap[q]_{\sim} .
$$

Proof. Let $p$ and $q$ be in $\beta S$ and let $A \in \cap[p+q]$. We show that $A \in \cap[p]+$ $\cap[q]$. To this end, we let $r \in[q]$ and show that $\{s \in S: A-s \in \cap[p]\} \in r$. Since $r \in[q]$ we have by assumption that $p+r \sim p+q$. Thus $A \in \cap[p+r]$. Pick, by Lemma 6.6, $f \in W(S)$ and $\delta>0$ such that $\operatorname{Range}(f) \subseteq\{x \in \mathbf{R}: 0 \leqslant x \leqslant 1\}$, $f^{\beta}(p+r)=0$, and $f^{-1}[[0, \delta)] \subseteq A$. Let $B=f^{-1}[[0, \delta / 2)]$. Then $B \in \cap[p+r]$ so $C=\{s \in S: B-s \in p\} \in r$. For $s \in S$, define $g_{s}$ by $g_{s}(t)=f(t+s)$. Then $g_{s} \in$ $W(S)$ and, for $\gamma>0, g_{s}^{-1}[[0, \gamma)]=f^{-1}[[0, \gamma)]-s$. Now given $s \in C$, we have $g_{s}^{-1}[[0, \delta / 2)] \in p \quad$ so $\quad g_{s}^{\beta}(p) \leqslant \delta / 2$. Thus $\left\{t \in S:\left|g_{s}(t)-g_{s}^{\beta}(p)\right|<\delta / 2\right\} \subseteq$ $g_{s}^{-1}[[0, \delta)]$ so, by Lemma $6.6, g_{s}^{-1}[[0, \delta)] \in \cap[p]$. Since $g_{s}^{-1}[[0, \delta)] \subseteq f^{-1}[[0, \delta)]-s$, we have $C \subseteq\left\{s \in S: f^{-1}[[0, \delta)]-s \in \cap[p]\right\}$. Since $f^{-1}[[0, \delta)] \subseteq A, C \subseteq\{s \in S$ : $A-s \in \cap[p]\}$ so $\{s \in S: A-s \in \cap[p]\} \in r$ as desired.

6.9 Lemma. Let $p, q \in \beta S$ and let $A \subseteq S$. If $A \in \cap[p+q]$, then $\{t \in S$ : $-t+A \in \cap[q]\} \in p$, where $-t+A=\{s \in S: t+s \in A\}$.

Proof. Let $A \in \cap[p+q]$, and pick, by Lemma 6.6, $f \in W(S)$ and $\delta>0$ such that $f^{\beta}(p+q)=0$, Range $(f) \subseteq\{x \in \mathbf{R}: 0 \leqslant x \leqslant 1\}$, and $f^{-1}[(0, \delta)] \subseteq A$. Let $B=$ $f^{-1}[[0, \delta / 3)]$. Then $B \in \bigcap[p+q]$ so, in particular, $C=\{s \in S: B-s \in p\} \in q$. 
Given $t \in S$, define $g_{t} \in W(S)$ by $g_{t}(s)=f(t+s)$. Then for $\gamma>0, g_{t}^{-1}[[0, \gamma)]=-t$ $+f^{-1}[[0, \gamma)]$. Note that if $g_{t}^{-1}[[0, \delta / 2)] \in q$, then $g_{t}^{\beta}(q) \leqslant \delta / 2$ so that $\{s \in S$ : $\left.\left|g_{t}(s)-g_{t}^{\beta}(q)\right|<\delta / 2\right\} \subseteq g_{t}^{-1}[[0, \delta)]$ and hence, by Lemma $6.6, g_{t}^{-1}[[0, \delta)] \in \cap[q]$. Thus it suffices to show, with $D=f^{-1}[[0, \delta / 2)]$, that $\{t \in S:-t+D \in q\} \in p$. (Because $\{t \in S:-t+D \in q\} \subseteq\{t \in S:-t+A \in \cap[q]\}$.) Suppose instead that $E=\{t \in S:-t+D \notin q\} \in p$. Let $t_{1} \in E$. Inductively pick

$$
s_{n} \in C \cap \bigcap_{k=1}^{n}\left(S \backslash\left(-t_{k}+D\right)\right) \text { and } t_{n+1} \in E \cap \bigcap_{k=1}^{n}\left(B-s_{k}\right) \text {. }
$$

Then, if $k \leqslant n$ we have $t_{k}+s_{n} \notin D$ so that $f\left(t_{k}+s_{n}\right) \geqslant \delta / 2$. Also, if $k<n$ then $t_{n}+s_{k} \in B$ so that $f\left(t_{n}+s_{k}\right) \leqslant \delta / 3$. Thus, thinning the sequences $\left\langle t_{n}\right\rangle_{n=1}^{\infty}$ and $\left\langle s_{n}\right\rangle_{n=1}^{\infty}$ so that all limits exist, we have $\lim _{k \rightarrow \infty} \lim _{n \rightarrow \infty} f\left(t_{n}+s_{k}\right) \leqslant \delta / 3$ while $\lim _{n \rightarrow \infty} \lim _{k \rightarrow \infty} f\left(t_{n}+s_{k}\right) \geqslant \delta / 2$ so that $f \notin W(S)$.

6.10 THEOREM. For all $p$ and $q$ in $\beta S$

$$
\cap[p+q]_{\sim} \subseteq \cap[p]_{\sim}+\cap[q]_{\sim} .
$$

Proof. Let $p, q$ and $r$ be in $\beta S$ and assume $q \sim r$. By Lemma 6.8 it suffices to show that $p+q \sim p+r$. Suppose instead we have $f \in W(S)$ such that $f^{\beta}(p+q) \neq$ $f^{\beta}(p+r)$ and let $\varepsilon=\left|f^{\beta}(p+q)-f^{\beta}(p+r)\right|$. Let

$$
A=\left\{s \in S:\left|f(s)-f^{\beta}(p+q)\right|<\varepsilon / 3\right\}
$$

and let

$$
B=\left\{s \in S:\left|f(s)-f^{\beta}(p+r)\right|\right\}<\varepsilon / 3 .
$$

By Lemma 6.6, $A \in \cap[p+q]$ and $B \in \cap[p+r]$. In particular $B \in p+r$. By Lemma 6.9, $\{t \in S:-t+A \in \cap[q]\} \in p$. Since $[q]=[r]$, we have $\{t \in S:-t+A$ $\in r\} \in p$. Let $C=\{s: B-s \in p\}$ and let $D=\{t \in S:-t+A \in r\}$. Let $t_{1} \in D$. Inductively let $s_{n} \in C \cap \cap_{k=1}^{n}\left(-t_{k}+A\right)$ and let $t_{n+1} \in D \cap \cap_{k=1}^{n}\left(B-s_{k}\right)$. Thinning the sequences $\left\langle t_{n}\right\rangle_{n=1}^{\infty}$ and $\left\langle s_{n}\right\rangle_{n=1}^{\infty}$ we get

$$
\left|\lim _{n \rightarrow \infty} \lim _{k \rightarrow \infty} f\left(t_{n}+s_{k}\right)-f^{\beta}(p+q)\right| \leqslant \varepsilon / 3
$$

and

$$
\left|\lim _{k \rightarrow \infty} \lim _{n \rightarrow \infty} f\left(t_{n}+s_{k}\right)-f^{\beta}(p+r)\right| \leqslant \varepsilon / 3,
$$

a contradiction.

We now know for $\Re=\left\{\cap[p]_{\sim}: p \in \beta S\right\}$, that $(\Re, e)$ is a Hausdorff weak compactification of $S$ and that $(\Re, \dot{+})$ is a left topological semigroup where $\cap[p] \dot{+} \cap[q]=\cap[p+q]$. We observe that if $S$ is commutative, then by Theorem 3.1, $[p+q]=[q+p]$ whenever $p, q \in \beta S$. Thus, if $S$ is commutative, $R$ is trivially a semitopological semigroup. We now proceed to show commutativity is not necessary for the conclusion.

6.11 TheOREM. Let $\Re=\left\{\cap[p]_{\sim}: p \in \beta S\right\}$. Then $\Re$ is a semitopological semigroup. 
Proof. Let $p \in \beta S$ and let $\mathscr{A}=\bigcap[p]$. We need only show $\rho_{\mathscr{Q}}$ is continuous. Suppose not and pick $q \in \beta S$ such that $\rho_{Q}$ is not continuous at $\cap[q]$, and pick $A \in \cap[q+p]\left(=\left(\rho_{\mathbb{Q}}(\cap[q])\right)\right)$ such that $\rho_{\mathbb{Q}}^{-1}\left[A^{*}\right]$ contains no neighborhood of $\cap[q]$. Pick $\delta>0$ and $f \in W(S)$ such that Range $(f) \subseteq\{x \in \mathbf{R}: 0 \leqslant x \leqslant 1\}, f^{\beta}(q+p)=0$, and $f^{-1}[[0, \delta)] \subseteq A$.

We show first that for all $D \in \cap[q]$ there is some $t \in D$ such that $\{s \in S$ : $f(t+s) \geqslant \delta / 2\} \in p$. Suppose instead we have $D \in \cap[q]$ such that for all $t \in D$, $\{s \in S: f(t+s)<\delta / 2\} \in p$. Since $D^{*}$ is not contained in $\rho_{\mathfrak{Q}}^{-1}\left[A^{*}\right]$, pick $r \in \beta S$ such that $D \in \cap[r]$ and $A \notin \cap[r+p]$. Now if $f^{\beta}(r+p)<\delta$, we would have, with $\mu=\delta-f^{\beta}(r+p), \quad\left\{s \in S:\left|f(s)-f^{\beta}(r+p)\right|<\mu\right\} \subseteq A$ and hence $A \in$ $\cap[r+p]$ by Lemma 6.6. Thus $f^{\beta}(r+p) \geqslant \delta$. Thus $\{s \in S: f(s)>2 \delta / 3\} \in r+p$. Let $B=\{s \in S: f(s)>2 \delta / 3\}$ and let $C=\{s \in S: B-s \in r\}$. Then $C \in p$. Let $s_{1} \in C$; and, inductively, let $t_{n} \in D \cap \cap_{k=1}^{n}\left(B-s_{k}\right)$; and let

$$
s_{n+1} \in C \cap \bigcap_{k=1}^{n}\left\{s \in S: f\left(t_{k}+s\right)<\delta / 2\right\} \text {. }
$$

Then, after thinning we have

$$
\lim _{n \rightarrow \infty} \lim _{k \rightarrow \infty} f\left(t_{n}+s_{k}\right) \leqslant \delta / 2
$$

while $\lim _{k \rightarrow \infty} \lim _{n \rightarrow \infty} f\left(t_{n}+s_{k}\right) \geqslant 2 \delta / 3$. This contradiction establishes the claim.

Let $E=f^{-1}[[0, \delta / 3)]$ and let $F=\{s \in S: E-s \in \cap[q]\}$. Then $E \in \cap[q+p]$ so, by Theorem 6.10, $F \in \cap[p]$ and hence $F \in p$. Pick $s_{1} \in F$. Inductively, $\bigcap_{k=1}^{n}\left(E-s_{k}\right) \in \cap[q]$ so pick $t_{n} \in \bigcap_{k=1}^{n}\left(E-s_{k}\right)$ such that $\left\{s \in S: f\left(t_{n}+s\right) \geqslant\right.$ $\delta / 2\} \in p$. Pick $s_{n+1} \in F \cap \cap_{k=1}^{n}\left\{s \in S: f\left(t_{k}+s\right) \geqslant \delta / 2\right\}$. Again, after thinning we obtain $\lim _{n \rightarrow \infty} \lim _{k \rightarrow \infty} f\left(t_{n}+s_{k}\right) \geqslant \delta / 2$ while $\lim _{k \rightarrow \infty} \lim _{n \rightarrow \infty} f\left(t_{n}+s_{k}\right) \leqslant \delta / 3$.

The next theorem says that $\left\{\cap[p]_{\sim}: p \in \beta S\right\}$ "is" $\omega S$.

6.12 THEOREM. Let $R=\left\{\cap[p]_{\sim}: p \in \beta S\right\}$. Then

(1) $e$ is a (continuous) homomorphism from $S$ to $\mathscr{R}$,

(2) $R$ is a compact Hausdorff semitopological semigroup,

(3) $e[S]$ in dense in $R$, and

(4) if $(T, \phi)$ satisfies (1) and (2), with $T$ replacing $\mathcal{G}$ and $\phi$ replacing $e$, there is a continuous homomorphism $\eta: \mathcal{R} \rightarrow T$ such that $\eta \circ e=\phi$.

Proof. Statements (1), (2) and (3) follow from Theorems 5.18, 6.7, 6.10 and 6.11. (Theorem 6.10 is needed to show that the hypotheses of Theorem 5.18 are satisfied.)

Let $(T, \phi)$ satisfy (1) and (2). We first show there is a continuous $\eta: R \rightarrow T$ such that $\eta \circ e=\phi$. By Lemma 6.1 it suffices to show for this that for each $p \in \beta S, \phi^{\beta}$ is constant on $[p]$. Suppose instead that we have $q \sim p$ with $\phi^{\beta}(q) \neq \phi^{\beta}(p)$. Since $T$ is completely regular, pick $f \in C(T)$ such that $f\left(\phi^{\beta}(q)\right) \neq f\left(\phi^{\beta}(p)\right)$. By Lemma 2.9, $f \in W(T)$. Consequently $f \circ \phi \in W(S)$. (Given $\left\langle t_{n}\right\rangle_{n=1}^{\infty}$ and $\left\langle s_{n}\right\rangle_{n=1}^{\infty}$ in $S$ such that $\lim _{n \rightarrow \infty} \lim _{k \rightarrow \infty} f \circ \phi\left(t_{n}+s_{k}\right)$ and $\lim _{k \rightarrow \infty} \lim _{n \rightarrow \infty} f \circ \phi\left(t_{n}+s_{k}\right)$ exist, we have

$$
\begin{aligned}
\lim _{n \rightarrow \infty} \lim _{k \rightarrow \infty} f\left(\phi\left(t_{n}+s_{k}\right)\right) & =\lim _{n \rightarrow \infty} \lim _{k \rightarrow \infty} f\left(\phi\left(t_{n}\right)+\phi\left(s_{k}\right)\right) \\
& =\lim _{k \rightarrow \infty} \lim _{n \rightarrow \infty} f\left(\phi\left(t_{n}\right)+\left(\phi\left(s_{k}\right)\right)=\lim _{k \rightarrow \infty} \lim _{n \rightarrow \infty} f\left(\phi\left(t_{n}+s_{k}\right)\right) .\right)
\end{aligned}
$$


But $f \circ \phi^{\beta}$ is a continuous extension of $f \circ \phi$ to $\beta S$ so $f \circ \phi^{\beta}=(f \circ \phi)^{\beta}$. But then $(f \circ \phi)^{\beta}(p) \neq(f \circ \phi)^{\beta}(q)$ so that $p \nsim q$, a contradiction.

To complete the proof, we show that $\eta$ is a homomorphism. We cannot invoke Lemma 6.2 because we do not know that $Q+\mathscr{R} \in \mathcal{R}$ whenever $Q$ and $\mathscr{B}$ are in $\mathscr{R}$ or that $\{\hat{s}: s \in S\} \subseteq \Re$. However, $\beta S$ satisfies the hypotheses of Lemma 6.2 so we have $\phi^{\beta}$ is a homomorphism. Since $\eta \circ \pi$ is a continuous extension of $\phi$, we have $\eta \circ \pi=\phi^{\beta}$. Thus, given $p$ and $q$ in $\beta S$, we have

$$
\begin{aligned}
\eta(\cap[p]+\cap[q]) & =\eta(\cap[p+q])=\eta \circ \pi(p+q) \\
& =\phi^{\beta}(p+q)=\phi^{\beta}(p)+\phi^{\beta}(q) \\
& =\eta \circ \pi(p)+\eta \circ \pi(q)=\eta(\cap[p])+\eta(\cap[q]) .
\end{aligned}
$$

The remark that $\mathscr{R}=\left\{\cap[p]_{\sim}: p \in \beta S\right\}$ “is" $\omega S$ is justified by the fact that, by Theorem 2.5, there is a continuous homomorphism $\psi: \omega S \rightarrow \Re$ such that $\psi \circ \omega_{S}=e$, and, by Theorem 6.12 , there is a continuous homomorphism $\eta: R \rightarrow \omega S$ such that $\eta \circ e=\omega_{S}$. Thus $\eta$ is an isomorphism and a homeomorphism leaving the image of $S$ "fixed".

The following theorem is our promised "internal" characterization of $\omega S$ as a space of filters. For the same reasons which are discussed before Theorem 5.11, we need to add conditions on $h$ to prevent an unnatural identification of points of $S$ with members of $R$.

6.13 THEOREM. Let $\Re$ be a set of filters on $S$. There exist an operation * on $\mathcal{R}$ and a function $h: S \rightarrow R$ (satisfying conditions (a)(i) and (ii) of Theorem 5.11) such that $(\Re, *, h)$ is $\omega S$ if and only if $\Re$ satisfies conditions (b), (c) and (d) of Theorem 6.7.

Proof. (Sufficiency) By Theorem 6.7, $\Re=\left\{\cap[p]_{\sim}: p \in \beta S\right\}$ so Theorem 6.12 applies.

(Necessity) By Theorem 5.11, $\Re$ is a quotient of $\beta S$. Thus by Corollary 5.9, the function $\pi: \beta S \rightarrow \Re$ defined by $\pi(p) \subseteq p$ is (well defined and) a quotient map. By condition (a)(i), for each $s \in S, s \in \cap h(s)$. That is, $h(s) \subseteq \hat{s}$. Then $h(s)=\pi(\hat{s})=$ $e(s)$ so $h=e$.

Define an equivalence relation $R$ on $S$ by $p R q$ if and only if $\pi(p)=\pi(q)$. It suffices to show that $p R q$ if and only if $p \sim q$. For then we get $R=\left\{\cap[p]_{\sim}\right.$ : $p \in \beta S\}$ and Theorem 6.7 applies. To this end, let $p, q \in \beta S$ and assume $p R q$. Suppose $p \nsim q$ and pick $f \in W(S)$ such that $f^{\beta}(p) \neq f^{\beta}(q)$. By Theorem 2.5, there exists $g \in C(\Re)$ such that $g \circ e=f$. Then $g \circ \pi$ is a continuous extension of $f$ to $\beta S$. $\left(\right.$ For $s \in S,(g \circ \pi)(\hat{s})=g(e(s))=f(s)$.) Thus $g \circ \pi=f^{\beta}$. Since $p R q$, then $f^{\beta}(p)=$ $g(\pi(p))=g(\pi(q))=f^{\beta}(q)$, contradiction. Now assume that $p \sim q$ and suppose that $\pi(p) \neq \pi(q)$. Pick $g \in C(\Re)$ such that $g(\pi(p)) \neq g(\pi(q))$. Define $f \in C(S)$ by $f(s)=g(e(s))$. Then, since $f$ extends continuously to $R, f \in W(S)$ by Theorem 2.5. But, as above $g \circ \pi=f^{\beta}$ so $f^{\beta}(p) \neq f^{\beta}(q)$, a contradiction.

For a topological space $X$ we denote by $C_{0}(X)$ and $C_{00}(X)$, respectively, these functions in $C(X)$ vanishing at infinity and those with compact support. 
6.14 THEOREM. The following statements are equivalent ( for any discrete semigroup $S)$.

(a) $\omega S$ is a topological compactification of $S$.

(b) $\omega_{S}: S \rightarrow \omega_{S}[S]$ is a homeomorphism.

(c) $C_{0}(S) \subseteq W(S)$.

(d) $C_{00}(S) \subseteq W(S)$.

(e) For all $r \in S, \chi_{\{r\}} \in W(S)$.

(f) There do not exist $r \in S$ and sequences $\left\langle t_{n}\right\rangle_{n=1}^{\infty}$ and $\left\langle s_{n}\right\rangle_{n=1}^{\infty}$ such that either

(i) $t_{n}+s_{k}=r$ and $t_{k}+s_{n} \neq r$ whenever $n<k$, or

(ii) $t_{n}+s_{k} \neq r$ and $t_{k}+s_{n}=r$ whenever $n<k$.

(g) $\omega_{S}$ is one-to-one.

(h) $\{\hat{s}: s \in S\} \subseteq\left\{\cap[p]_{\sim}: \rho \in \beta S\right\}$.

Proof. We represent $\omega S$ as $\mathscr{R}=\left\{\cap[p]_{\sim}: p \in \beta S\right\}$ and $\omega_{S}$ by $e$ (which we are justified in doing by Theorem 6.12).

That (a) implies (b) is trivial.

By Lemma III.4.4 of [2], statements (b) and (c) are equivalent.

That (c) implies (d) and (d) implies (e) are trivial.

To see that (e) implies (f), suppose we have such $r,\left\langle t_{n}\right\rangle_{n=1}^{\infty}$ and $\left\langle s_{n}\right\rangle_{n=1}^{\infty}$. If alternative (i) holds, then

$$
\lim _{n \rightarrow \infty} \lim _{k \rightarrow \infty} \chi_{\{r\}}\left(t_{n}+s_{k}\right)=1
$$

while $\lim _{k \rightarrow \infty} \lim _{n \rightarrow \infty} \chi_{\{r\}}\left(t_{n}+s_{k}\right)=0$. If alternative (ii) holds, then

$$
\lim _{n \rightarrow \infty} \lim _{k \rightarrow \infty} \chi_{\{r\}}\left(t_{n}+s_{k}\right)=0
$$

while $\lim _{k \rightarrow \infty} \lim _{n \rightarrow \infty} \chi_{\{r\}}\left(t_{n}+s_{k}\right)=1$. In either case we have $\chi_{\{r\}} \notin W(S)$, and we have the contrapositive.

To see that (f) implies (e) suppose we have $r \in S$ such that $\chi_{\{r\}} \notin W(S)$. Pick sequences $\left\langle t_{n}\right\rangle_{n=1}^{\infty}$ and $\left\langle s_{k}\right\rangle_{k=1}^{\infty}$ such that

$$
\lim _{n \rightarrow \infty} \lim _{k \rightarrow \infty} \chi_{\{r\}}\left(t_{n}+s_{k}\right) \neq \lim _{k \rightarrow \infty} \lim _{n \rightarrow \infty} \chi_{\{r\}}\left(t_{n}+s_{k}\right) .
$$

If $\lim _{n \rightarrow \infty} \lim _{k \rightarrow \infty} \chi_{\{r\}}\left(t_{n}+s_{k}\right)=1$, we have (after thinning the sequences) that condition (f)(i) holds. Similarly, if $\lim _{n \rightarrow \infty} \lim _{k \rightarrow \infty} \chi_{\{r\}}\left(t_{n}+s_{k}\right)=0$ we obtain condition (f)(ii). We have the contrapositive.

To see that (e) implies (g), let $s, r \in S$ and assume $e(s)=e(r)$. Then $\chi_{\{r\}} \in W(S)$ so by Theorem 2.5 there exists $f \in C(\mathscr{R})$ such that $f \circ e=\chi_{\{r\}}$. Then $1=\chi_{\{r\}}(r)=$ $f(e(r))=f(e(s))=\chi_{\{r\}}(s)$ so $s=r$.

To see that (g) implies (a) note first that by Theorem $6.7,(G R, e)$ is a Hausdorff weak compactification of $S$. By assumption, $e$ is one-to-one. By Theorem 5.11, $e$ is an open map.

Finally (a) and (h) are equivalent by Corollary 5.12 .

We observe that it is easy to obtain discrete $S$ failing to satisfy the conditions of Theorem 6.14. For example, let $S=\mathbf{N} \cup\{0\}$ and for $n \in \mathbf{N}$ let $s_{n}=2 n+1$ and $t_{n}=2 n$. Define a commutative operation $*$ on $S$ by $s_{n} * t_{m}=0$ if $n \geqslant m, s_{n} * t_{m}=1$ if $n<m$, and all other products equal 0 . In particular, $0 * 0=1 * 0=1 * 1=0$. Then $*$ 
is associative since for all $x, y, z \in S, x *(y * z)=(x * y) * z=0$. With $r=0$ or $r=1$, we have that condition (f) of Theorem 6.14 is violated. (We are indebted to the referee for pointing out this very neat example.)

We now proceed to apply our results to a determination of the size of $\omega S$ under certain conditions of $S$.

6.15 Lemma. Let $A \subseteq S$. There exist $p$ and $q$ in $\beta S \backslash S$ such that $A \in p+q$ if and only if there exist one-to-one sequences $\left\langle t_{n}\right\rangle_{n=1}^{\infty}$ and $\left\langle s_{n}\right\rangle_{n=1}^{\infty}$ in $S$ such that $\left\{t_{n}+s_{k}\right.$ : $k \leqslant n\} \subseteq A$.

Proof. (Necessity) Pick such $p$ and $q$. Let $B=\{s \in S: A-s \in p\}$. Then $B \in q$ and, since $q \notin e[S], B$ is infinite. Let $\left\langle s_{n}\right\rangle_{n=1}^{\infty}$ be a one-to-one sequence in $B$. For each $n$, we have $\cap_{k=1}^{n}\left(A-s_{k}\right) \in p$ and is hence infinite. Pick $t_{n} \in \cap_{k=1}^{n}\left(A-s_{k}\right)$ such that $t_{n} \notin\left\{t_{k}: k<n\right\}$.

(Sufficiency) Pick $q \in \beta S \backslash S$ such that $\left\{s_{n}: n \in \mathbf{N}\right\} \in q$. For each $n$, let $B_{n}=\left\{t_{m}\right.$ : $m \geqslant n\}$. Pick $p \in \beta S \backslash S$ such that $\left\{B_{n}: n \in \mathbf{N}\right\} \subseteq p$. We claim that $\left\{s_{n}: n \in \mathbf{N}\right\} \subseteq$ $\{s: A-s \in p\}$ and hence that $A \in p+q$ as desired. Given $s_{n}, t_{m}+s_{n} \in A$ whenever $m \geqslant n$ so $B_{n} \subseteq A-s_{n}$ and hence $A-s_{n} \in p$.

6.16 Lemma. Let $p \in(\beta S \backslash S) \backslash \mathrm{cl}_{\beta S}((\beta S \backslash S)+(\beta S \backslash S))$. Then for each $q \in$ $\beta S \backslash\{p\}$ there exists $f \in W(S)$ such that $\operatorname{Range}(f)=\{0,1\}$ and $f^{\beta}(p)=1$ and $f^{\beta}(q)=0$. Consequently $[p]=\{p\}$.

Proof. Pick $A \in p$ such that $\bar{A} \cap((\beta S \backslash S)+(\beta S \backslash S))=\varnothing$. Pick $B \in p$ such that $B \notin q$. Let $f=\chi_{A \cap B}$. Then $f^{\beta}(p)=1$ and $f^{\beta}(q)=0$ so it suffices to show that $f \in W(S)$. Suppose, instead, we have sequences $\left\langle t_{n}\right\rangle_{n=1}^{\infty}$ and $\left\langle s_{n}\right\rangle_{n=1}^{\infty}$ such that $\lim _{k \rightarrow \infty} \lim _{n \rightarrow \infty} f\left(t_{n}+s_{k}\right)=1$ and $\lim _{n \rightarrow \infty} \lim _{k \rightarrow \infty} f\left(t_{n}+s_{k}\right)=0$. We may assume by thinning that $\left\langle t_{n}\right\rangle_{n=1}^{\infty}$ and $\left\langle s_{n}\right\rangle_{n=1}^{\infty}$ are one-to-one and that, when $n \geqslant k$, $f\left(t_{n}+s_{k}\right)=1$. But then $\left\{t_{n}+s_{k}: n \geqslant k\right\} \subseteq A$ so, by Lemma $6.15, \bar{A} \cap((\beta S \backslash S)+$ $(\beta S \backslash S)) \neq \varnothing$.

6.17 TheOrem. Let $S$ be infinite and assume there exists $A \subseteq S$ such that (i) $\operatorname{card}(A)=\operatorname{card}(S)$ and (ii) there do not exist one-to-one sequences $\left\langle t_{n}\right\rangle_{n=1}^{\infty}$ and $\left\langle s_{n}\right\rangle_{n=1}^{\infty}$ such that $\left\{t_{n}+s_{k}: k \leqslant n\right\} \subseteq A$. Then $\operatorname{card}(\omega S)=2^{2^{\operatorname{card}(S)} \text {. }}$

Proof. As is well known, the number of ultrafilters on a set $X$ is $2^{2^{\operatorname{card}(X)}}$. (See the notes to Chapter 9 of [7] for the origins of this fact.) Thus $\operatorname{card}(\omega S) \leqslant \operatorname{card}(\beta S)=$ $2^{2^{\operatorname{card}(S)}}$. On the other hand, we have, by Lemma 6.15 , that $\bar{A} \cap((\beta S \backslash S)+(\beta S \backslash S))$ $=\varnothing$. Thus, by Lemma 6.16 , for each $p \in \bar{A},[p]_{\sim}=\{p\}$. Therefore $\operatorname{card}(\omega S) \geqslant$ $\operatorname{card}(\bar{A})=2^{2^{\operatorname{card}(S)}}$.

6.18 CoRollaRY. If $S$ is any of $(\mathbf{N},+),(\mathbf{N}, \cdot),(\mathbf{Z},+)$ or $(\mathbf{Z}, \cdot)$, then $\operatorname{card}(\omega S)=2^{c}$, where $c=\operatorname{card}(\mathbf{R})$.

It would have been convenient at times if we could show that, for all $p$ and $q$ in $\beta S, \cap[p]+\cap[q]=\cap[p+q]$. We conclude by showing that this need not be true.

6.19 TheOREM. There exist $p$ and $q$ in $\beta \mathbf{N}$ such that $\cap[p+q] . \neq \cap[p]$. $+\cap[q]_{\sim}$. 
Proof. Let $A=\left\{2^{2 n}: n \in \mathbf{N}\right\}$ and let $B=\left\{2^{2 n+1}: n \in \mathbf{N}\right\}$ and pick $p$ and $q$ in $\beta \mathbf{N} \backslash \mathbf{N}$ such that $A \in p$ and $B \in q$. Then by Lemma 6.15,

$$
\bar{A} \cap((\beta \mathbf{N} \backslash \mathbf{N})+(\beta \mathbf{N} \backslash \mathbf{N}))=\varnothing \quad \text { and } \quad \bar{B} \cap((\beta \mathbf{N} \backslash \mathbf{N})+(\beta \mathbf{N} \backslash \mathbf{N}))=\varnothing .
$$

Thus by Lemma $6.16,[p]=\{p\}$ and $[q]=\{q\}$. Suppose that $\cap[p]+\cap[q]=$ $\cap[p+q]$. Then $p+q=\cap[p+q]$ and hence

$$
\cap[q+p]=\cap[q]+\cap[p]=\cap[\dot{p}]+\cap[q]=\cap[p+q]=p+q,
$$

hence $p+q=q+p$ (since $p+q=\cap[q+p] \subseteq q+p)$. Let $C=\left\{2^{2 n}+2^{2 k+1}\right.$ : $k<n\}$ and let $D=\left\{2^{2 n}+2^{2 k+1}: k>n\right\}$. Then $A \subseteq\{x \in \mathbf{N}: D-x \in q\}$. (For given $n \in \mathbf{N},\left\{2^{2 k+1}: k>n\right\} \subseteq D-2^{2 n}$ and $\left\{2^{2 k+1}: k>n\right\} \in q$ since $q \in \beta \mathbf{N} \backslash \mathbf{N}$.) Thus $D \in q+p$. Similarly, $C \in p+q$. Since $D \cap C=\varnothing, p+q \neq q+p$, a contradiction.

ACKNOWLEDGEMENT. This paper has benefited from a very helpful and constructive referee's report. We want to acknowledge our debt to the referee.

\section{REFERENCES}

1. J. F. Berglund, On extending almost periodic functions, Pacific J. Math. 33 (1970), 281-289.

2. J. F. Berglund and K. H. Hofmann, Compact semitopological semigroups and weakly almost periodic functions, Lecture Notes in Math., vol. 42, Springer-Verlag, Berlin, 1967.

3. J. F. Berglund, H. D. Junghenn and P. Milnes, Compact right topological semigroups and generalizations of almost periodicity, Lecture Notes in Math., vol. 663, Springer-Verlag, Berlin, 1978.

4. R. B. Burckel, Weakly almost periodic functions on semigroups, Gordon \& Breach, New York, 1970.

5. W. W. Comfort, Ultrafilters-some old and some new results, Bull. Amer. Math. Soc. 83 (1977), $417-455$.

6. N. Dunford and J. T. Schwartz, Linear operators. I, Wiley, New York, 1964.

7. L. Gillman and M. Jerison, Rings of continuous functions, Van Nostrand, Princeton, N. J., 1960.

8. E. Hewitt and K. A. Ross, Abstract harmonic analysis. I, Springer-Verlag, New York, 1963.

9. N. Hindman, On density, translates and pairwise sums of integers, J. Combin. Theory Ser. A 33 (1982), 147-157.

10. Sums equal to products in $\beta \mathbf{N}$, Semigroup Forum 21 (1980), 221-255.

11. Ultrafilters and combinatorial number theory, M. B. Nathanson (ed.), Number Theory, Carbondale 1979 Proceedings, Lecture Notes in Math., vol. 751, Springer-Verlag, Berlin, 1979, pp. $119-184$.

12. K. H. Hofmann and P. S. Mostert, Elements of compact semigroups, Merrill, Columbus, Ohio, 1966.

13. J. D. Lawson, Additional notes on continuity in semitopological semigroups, Semigroup Forum 12 (1976), 265-280.

14. Joint continuity in semitopological semigroups, Illinois J. Math. 18 (1974), 275-285.

15. K. deLeeuw and I. Glicksberg, Applications of almost periodic compactifications, Acta Math. 105 (1961), 63-97.

Department of Mathematics, Virginia Commonwealth University, Richmond, Virginia 23284

Department of Mathematics, Howard University, Washington, D.C. 20059 